NASA Contractor Report 191471

ICASE Report No. 93-25
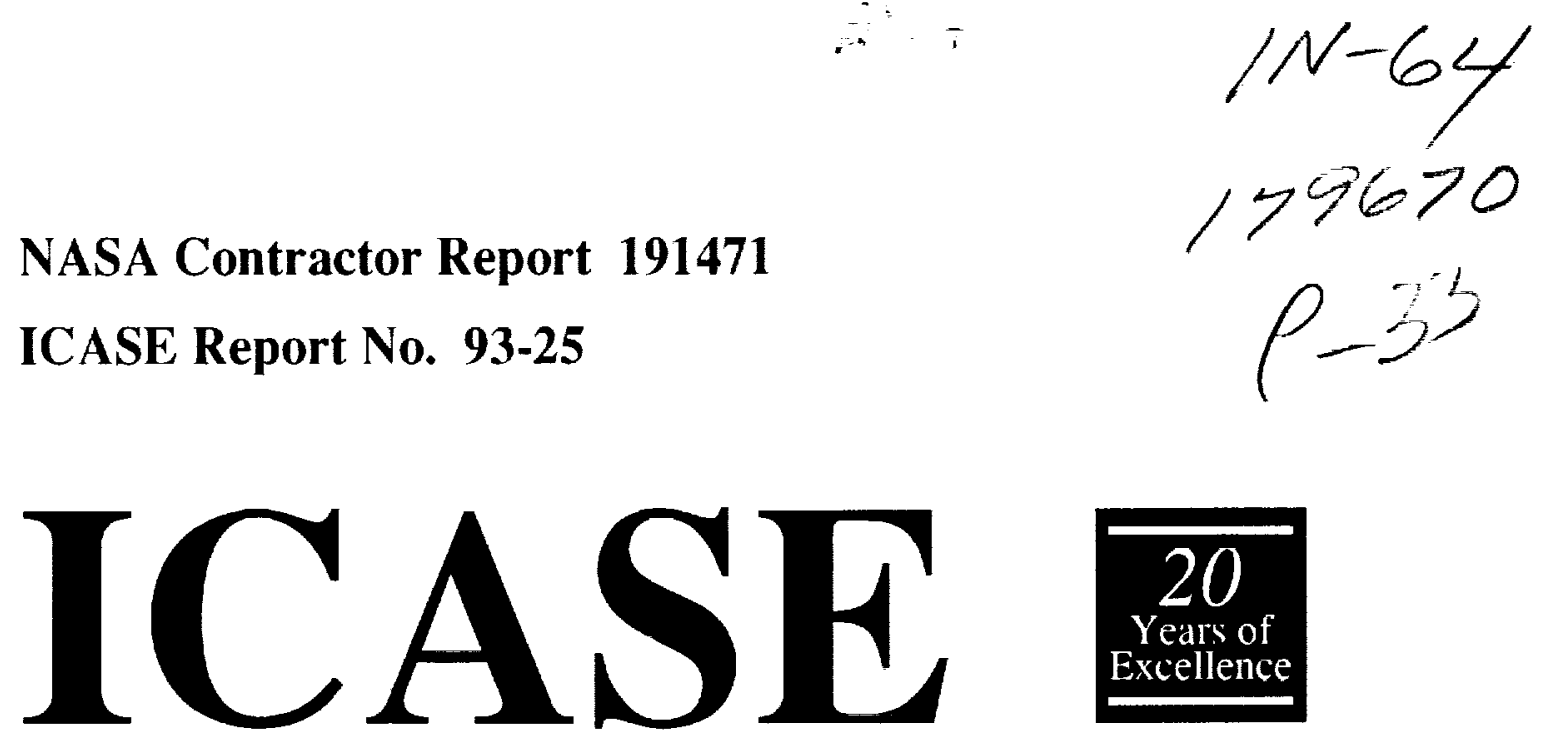

FINITE DIFFERENCE SCHEMES FOR LONG-TIME INTEGRATION

\title{
Zigo Haras
}

Shlomo Ta'asan

$\begin{array}{lll}N & & \\ + & & 0 \\ 0 & 0 & 0 \\ 0 & 0 & 0 \\ 1 & 0 & 0 \\ j & 0 & \frac{1}{2} \\ 2 & 5 & 0\end{array}$

NASA Contract Nos. NAS 1-19480, NAS1-18605

June 1993

Institute for Computer Applications in Science and Engineering NASA Langley Research Center Hampton, Virginia 23681-0001

Operated by the Universities Space Research Association

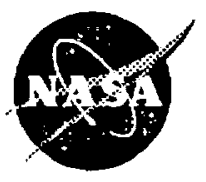

National Aeronautics and

Space Administration

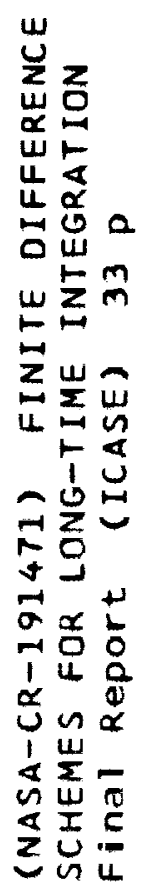


$+1$ 


\title{
Finite difference schemes for long-time integration
}

\author{
Zigo Haras and Shlomo Ta'asan * \\ Department of Applied Mathematics and Computer Science \\ The Weizmann Institute of Science \\ and \\ Institute for Computer Applications in Science and Engineering
}

\begin{abstract}
Finite difference schemes for the evaluation of first and second derivatives are presented. These second order compact schemes were designed for long-time integration of evolution equations by solving a quadratic constrained minimization problem. The quadratic cost function measures the global truncation error while taking into account the initial data. The resulting schemes are applicable for integration times fourfold, or more, longer than similar previously studied schemes. A similar approach was used to obtain improved integration schemes.
\end{abstract}

"This research was made possible in part by funds granted to the second author through a fellowship program sponsored by the Charles H. Revson Foundation and in part by the National Aeronautics and Space Administration under NASA Contract No. NAS1-19480 and NAS1-18605 while the authors were in residence at ICASE, NASA Langley Research Center, Hampton, Va 23681 


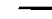




\section{Introduction}

The simulation of hyperbolic partial differential equations often requires long-time integration. The physical phenomena described by these equations typically possess a range of space and time scales, turbulent fluid flow is a common example. Accurate numerical simulation of this type of processes requires proper representation of all the relevant physical scales in the numerical model. These requirements lead recently to new interest in Pade approximations also known as compact finite difference schemes [7].

Compact finite difference schemes had long been known and used in numerical analysis $[1,2,3]$. They offer a means of obtaining high order approximations to differential operators using narrow stencils. This is achieved by treating the sought derivatives as unknowns and solving a system of equations for them. Typically, the resulting matrices are tridiagonal or pentadiagonal, which can be efficiently solved .

In [7] a class of highly accurate compact schemes for first, second and higher derivatives were presented and analyzed. A notion of resolving efficiency was introduced which should measure the accuracy with which the finite difference approximation represents the exact solution over the full range of length scales that can be realized on a given grid. This criterion was then used to compare various schemes and motivated the design of a new class of schemes, the so-called schemes with spectral like resolution. These are fourth order pentadiagonal systems with seven points stencils. Their improved resolution characteristics were obtained by giving up on high formal accuracy; instead, requiring that the symbol of the discrete difference operator should agree with the differential operator at three prescribed high frequencies. However, the resolving efficiency is a too crude measure as it assumes that all frequencies occur with similar magnitude in the initial data. In the present paper it is shown that for problems with various initial conditions these schemes are far from optimal.

A different measure for evaluating finite difference schemes is the $L_{2}$ norm of the local truncation error. This measure which takes into account the Fourier components present in the solution and their amplitude was applied in [9] to design explicit time marching schemes (i.e., discretizing time and space simultaneously) by solving analytically constrained minimization problems with quadratic cost. This error measure seems more adequate for comparing difference schemes. However, the simultaneous treatment of time and space results in very complex optimization problems. The generalization of the approach of [9] to problems in higher dimensions requires solving nonlinear constrained optimization over a large set of parameters. It is also hard to apply that approach to compact schemes. This complexity and the use of analytic rather than numerical methods makes the suggested approach impractical.

In [4] a heuristic derivation was done by minimizing the weighted error (in the Fourier space) of the discrete and continuous operators.

The present paper uses the same cost function as [9] with several important differences. First, improved bounds on the truncation error were derived. These enabled us to treat time and space discretizations separately. This greatly simplifies the minimization problem by reducing it to two lower dimensional problems. Further simplification is obtained by optimizing each partial derivative separately, instead of approximating the whole differential operator as was done in [9]. These reductions of problem complexity resulted in a simple and general approach to synthesis of discretization schemes. It enabled us to design highly accurate compact difference schemes and integration formulas for various operators and initial data. The resulting second 
order approximations proved to be robust to perturbations in the spectrum of the initial data, exhibiting :resolution superior to other known schemes.

The organization of the paper is as follows. In Section 2 Fourier analysis is used to obtain bounds on the truncation error. In section 3 approximations to derivatives are presented, for the first and second derivatives and first derivative at mid cell points. Appendices A-C list coefficients for these derivatives for various stencils and initial conditions. Improved time integration schemes are developed in section 4, and their coefficients are listed at Appendix D. Section 5 discusses generalization of the present approach to more complex problems. Numerical results are presented in section 6 . Concluding remarks are made in section 7 .

\section{Bounds on the truncation error}

The application of Fourier analysis for the design and evaluation of finite difference schemes can be found in many sources, e.g., $[9,10]$. In [12] the use of Fourier analysis in the numerical approximation of hyperbolic problems is extensively discussed.

In the following section bounds on the $\mathrm{L}_{2}$ norm of the error in the discrete solution are derived, accounting for the effect of discretization both in space and time. These estimates are used in subsequent sections to design highly accurate schemes.

Consider a linear constant coefficient partial differential equation with periodic boundary conditions of the form :

$$
\begin{aligned}
\frac{\partial u}{\partial t} & =L u \\
u(x, 0) & =u_{0}(x)
\end{aligned}
$$

Further assume that the solution of equation (2.2) does not grow in time.

The discrete analog of this equation can be written as :

$$
\begin{aligned}
u_{h}^{n+1} & =P(h, \Delta t) u_{h}^{n} \\
u_{h}^{0} & =u_{0}
\end{aligned}
$$

where $\mathrm{h}$ is the meshsize in space, and $P(h, \Delta t)$ is a stable finite difference approximation.

We would like to bound the $L_{2}$ norm of the error in the discrete solution, for the initial value $u_{0}$, given by :

$$
e^{2}\left(n \Delta t ; u_{0}\right)=\left\|u(n \Delta t)-u_{h}^{n}\right\|^{2}=\left\|e^{L n \Delta t} u_{0}-P^{n}(h, \Delta t) u_{0}\right\|^{2}
$$

In the sequel $t$ will be used instead of $n \Delta t$ to simplify notation. The Fourier transform of eq. (2.5) yields

$$
\begin{gathered}
\int_{-\frac{\pi}{h}}^{\frac{\pi}{h}}\left|e^{L(\omega h) t}-\hat{P}^{n}(\omega h)\right|^{2}\left|\hat{u}_{0}(\omega h)\right|^{2} d \omega \leq \\
\int_{-\frac{\pi}{h}}^{\frac{\pi}{h}}\left(\left|e^{L(\omega h) t}-e^{L^{h}(\omega h) t}\right|+\left|e^{L^{h}(\omega h) t}-\hat{P}^{n}(\omega h)\right|\right)^{2}\left|\hat{u}_{0}(\omega h)\right|^{2} d \omega
\end{gathered}
$$

Where $L^{h}$ is the discrete operator approximating $L$, and $\hat{L}, \hat{L}^{h}$ are their corresponding symbols. Thus, the space and time discretizations errors can be bounded separately. 
Denote $\hat{L}(\omega h)=\hat{L}_{R}(\omega h)+\hat{L}_{I}(\omega h)$ for the real and imaginary parts of $\hat{L}(\omega h)$, respectively; and use a similar decomposition for $\hat{L}^{h}(\omega h)$. Then

$$
e^{\hat{L}(\omega h) t}-e^{\hat{L}^{h}(\omega h) t}=e^{\hat{L}(\omega h) t}\left(1-e^{\left(\hat{L}_{I}^{h}(\omega h)-\hat{L}_{I}(\omega h)\right) t} e^{\left(\hat{L}_{R}^{h}(\omega h)-\hat{L}_{R}(\omega h)\right) t}\right)
$$

The assumption that the solution does not grow in time implies that

$$
\left|e^{\mathcal{L}(\omega h) t}\right| \leq 1 \quad t \geq 0
$$

Assume that the discrete solution does not grow in time, either. That is

$$
\left|e^{\hat{L}^{h}(\omega h) t}\right| \leq 1
$$

For real numbers $\theta, \alpha$ with $\alpha<0$, simple geometric considerations yield the following bound

$$
\left|1-e^{i \theta} e^{\alpha}\right| \leq|\theta|+\left|1-e^{\alpha}\right|
$$

Combining bounds $(2.9)$ and (2.11) and assuming $\hat{L}_{R}^{h}(\omega h)-\hat{L}_{R}(\omega h)<0$ results in :

$$
\begin{aligned}
\left|e^{\hat{L}(\omega h) t}-e^{\hat{L}^{h}(\omega h) t}\right| & \leq\left|\hat{L}_{I}^{h}(\omega h)-\hat{L}_{I}(\omega h)\right| t+\left|1-e^{\left(\hat{L}_{R}^{h}(\omega h)-\hat{L}_{R}(\omega h)\right) t}\right| \\
& \leq\left|\hat{L}_{I}^{h}(\omega h)-\hat{L}_{I}(\omega h)\right| t+\left|\hat{L}_{R}^{h}(\omega h)-\hat{L}_{R}(\omega h)\right| t
\end{aligned}
$$

If $\hat{L}_{R}^{h}(\omega h)-\hat{L}_{R}(\omega h)>0$, this bound can be obtained using the same argument when factoring $e^{L^{h}(\omega h) t}$ in (2.8).

Denote by $\tilde{e}\left(t ; u_{0}\right)$ the error due to spatial discretization only, when the initial data is $u_{0}$. For a final time $T$, using (2.13) yields :

$$
\tilde{e}^{2}\left(T ; u_{0}\right) \leq T^{2} \int_{-\frac{\pi}{h}}^{\frac{\pi}{h}}\left(\left|\hat{L}_{I}^{h}(\omega h)-\hat{L}_{I}(\omega h)\right|+\left|\hat{L}_{R}^{h}(\omega h)-\hat{L}_{R}(\omega h)\right|\right)^{2}\left|\hat{u}_{0}(\omega h)\right|^{2} d \omega
$$

Therefore, a difference scheme minimizing the integral (2.14) with respect to initial value $u_{0}$ will better resolve, in the $\mathrm{L}_{2}$ norm sense, the frequencies occurring in the solution.

The time integration operator satisfies :

$$
e^{\hat{L}^{h}(\omega h) n \Delta t}-\hat{P}^{n}(\omega h)=\left(e^{L^{h}(\omega h) \Delta t}-\hat{P}(\omega h)\right) \sum_{j=1}^{n-1} e^{\hat{L}^{h}(\omega h) j \Delta t} \hat{P}^{n-1-j}(\omega h)
$$

Under the previous assumptions

$$
\begin{aligned}
\left|e^{L^{h}(\omega h) \Delta t}\right| & \leq 1 \\
|\hat{P}(\omega h)| & \leq 1
\end{aligned}
$$

Therefore

$$
\left|\sum_{j=1}^{n-1} e^{\hat{L}^{h}(\omega h) j \Delta t} \hat{P}^{n-1-j}(\omega h)\right| \leq C n
$$

where $C=O(1)$. Hence

$$
\left|e^{\hat{L}^{h}(\omega h) t}-\hat{P}^{n}(\omega h)\right| \leq \frac{C T}{\Delta t}\left|e^{\hat{L}^{h}(\omega h) \Delta t}-\hat{P}(\omega h)\right|
$$


Denote by $\bar{e}\left(t ; u_{0}\right)$ the error due to time discretization only, when the initial value is $u_{0}$. For a final time $T$, the bound (2.19) implies :

$$
\bar{e}^{2}\left(T, u_{0}\right) \leq\left(\frac{C T}{\Delta t}\right)^{2} \int_{-\frac{\pi}{h}}^{\frac{\pi}{h}}\left|e^{\hat{L}^{h}(\omega h) \Delta t}-\hat{P}(\omega h)\right|^{2}\left|\hat{u}_{0}(\omega h)\right|^{2} d \omega
$$

Combining of these estimates yields a bound on the $\mathrm{L}_{2}$ norm of the global truncation error :

$e^{2}\left(T ; u_{0}\right) \leq T^{2} \int_{-\frac{\pi}{h}}^{\frac{\pi}{h}}\left[\left|\hat{L}_{I}^{h}(\omega h)-\hat{L}_{I}(\omega h)\right|+\left|\hat{L}_{R}^{h}(\omega h)-\hat{L}_{R}(\omega h)\right|+\frac{C}{\Delta t} \mid e^{\hat{L}^{h}(\omega h) \Delta t}-\hat{P}(\omega h)\right]^{2}\left|\hat{u}_{0}(\omega h)\right|^{2} d \omega$

These estimates will be used in the following sections to design improved compact schemes and integration formulas.

\section{Approximating derivatives}

\subsection{Approximation of the first derivative}

Consider a uniformly spaced mesh whose nodes are indexed by $i$ and its meshsize is given by $h=\frac{1}{N}$, where $N+1$ is the number of grid points. The variable at node $i$ is $x_{i}=i h$ and the function value at the nodes, $f_{i}=f\left(x_{i}\right)$, are given for $0 \leq i \leq N$. An approximation $f_{i}^{\prime}$ to the first derivative $\frac{d f}{d x}\left(x_{i}\right)$ should be computed as a linear combination of the function values at neighboring grid points. Compact finite difference schemes regard the approximation $f_{i}^{\prime}$ as unknown and a system of equations is solved to approximate the first derivative at all nodes, simultaneously. Thus, unlike in finite differences, the derivative at node $i$ depends on function values at all other nodes.

Following [7] we use approximations of the the form :

$$
\beta f_{i-2}^{\prime}+\alpha f_{i-1}^{\prime}+f_{i}^{\prime}+\alpha f_{i+1}^{\prime}+\beta f_{i+2}^{\prime}=c \frac{f_{i+3}-f_{i-3}}{6 h}+b \frac{f_{i+2}-f_{i-2}}{4 h}+a \frac{f_{i+1}-f_{i-1}}{2 h}
$$

A second order approximation can be obtained by adding a constraint that the Taylor expansion on both sides should agree until the second order term. The following relation must hold :

$$
a+b+c=1+2 \alpha+2 \beta
$$

Higher order schemes may be obtained by further matching the next terms in the expansion [7]. However, in this paper merely second order accuracy is enforced.

The symbol of the differentiation operator is given by :

$$
\hat{L}(\omega h)=i \omega h
$$

Whereas the symbol of the discrete approximation (3.1) is

$$
\hat{L}^{h}(\omega h)=i \frac{a \sin (\omega h)+\frac{b}{2} \sin (2 \omega h)+\frac{c}{3} \sin (3 \omega h)}{1+2 \alpha \cos (\omega h)+2 \beta \cos (2 \omega h)}
$$


In view of the bound (2.14), define the following constrained minimization problem which its solution should yield a compact scheme with improved resolution properties :

$$
\min _{a, b, c, \alpha, \beta} \int_{-\frac{\pi}{h}}^{\frac{\pi}{h}}\left|\hat{L}^{h}(\omega h)-\hat{L}(\omega h)\right|^{2}|\hat{u}(\omega h)|^{2} d \omega
$$

under the constraint

$$
a+b+c=1+2 \alpha+2 \beta
$$

where $\hat{L}(\omega h)$ and $\hat{L}^{h}(\omega h)$ are given by (3.3) and (3.4), respectively.

Although the problem was formulated as a constrained minimization problem, it can be transformed by substitution to an unconstrained minimization problem on a reduced set of parameters. Moreover, setting some of the parameters to zero further reduces the dimension of the problem. Since tridiagonal systems of equations are more amenable to numerical solution than pentadiagonal ones, setting $\beta=0$ seems a plausible choice. Similar considerations might suggest using a narrower stencil obtained by setting $c=0$, as well. All those possibilities are presented in Section 6, and several sets of coefficients for different initial data are listed in Appendix A.

\subsection{Approximation of the second derivative}

The derivation of compact schemes for the second derivative proceeds in an analogous way to the first derivative. The starting point is an approximation of the form

$\beta f_{i-2}^{\prime \prime}+\alpha f_{i-1}^{\prime \prime}+f_{i}^{\prime \prime}+\alpha f_{i+1}^{\prime \prime}+\beta f_{i+2}^{\prime \prime}=c \frac{f_{i+3}-2 f_{i}+f_{i-3}}{9 h^{2}}+b \frac{f_{i+2}-2 f_{i}+f_{i-2}}{4 h^{2}}+a \frac{f_{i+1}-2 f_{i}+f_{i-1}}{h^{2}}$

where $f_{i}^{\prime \prime}$ is the approximation to the second derivative at node $i$. Matching the Taylor series coefficients on both sides of (3.7) yields condition (3.2) for the second order accuracy.

The symbol of the second derivative is given by :

$$
\hat{L}(\omega h)=-\omega^{2} h^{2}
$$

The symbol of the discrete approximation (3.7) is :

$$
\hat{L}^{h}(\omega h)=-\frac{2 a(\cos (\omega h)-1)+\frac{b}{2}(\cos (2 \omega h)-1)+\frac{9 c}{2}(\cos (3 \omega h)-1)}{1+2 \alpha \cos (\omega h)+2 \beta \cos (2 \omega h)}
$$

The constrained minimization problem which solution is the sought scheme can be formulated as :

$$
\min _{a, b, c, \alpha, \beta} \int_{-\frac{\pi}{h}}^{\frac{\pi}{h}}\left|\hat{L}^{h}(\omega h)-\hat{L}(\omega h)\right|^{2}|\hat{u}(\omega h)|^{2} d \omega
$$

under the constraint

$$
a+b+c=1+2 \alpha+2 \beta
$$

Now, however, $\hat{L}(\omega h)$ and $\hat{L}^{h}(\omega h)$ are given by (3.8) and (3.9), respectively. 


\subsection{Approximating first derivative on a cell centered mesh}

The approximation of the first derivative at the cell centered mesh is of the form :

$$
\beta f_{i-2}^{\prime}+\alpha f_{i-1}^{\prime}+f_{i}^{\prime}+\alpha f_{i+1}^{\prime}+\beta f_{i+2}^{\prime}=c \frac{f_{i+\frac{5}{2}}-f_{i-\frac{5}{2}}}{5 h}+b \frac{f_{i+\frac{3}{2}}-f_{i-\frac{3}{2}}}{3 h}+a \frac{f_{i+\frac{1}{2}}-f_{i-\frac{1}{2}}}{h}
$$

The second order of the approximation is guaranteed by condition (3.2).

The symbol of the differentiation operator is :

$$
\hat{L}(\omega h)=i \omega h
$$

While the symbol of the discrete approximation (3.12) is :

$$
\hat{L}^{h}(\omega h)=i \frac{2 a \sin \left(\frac{\omega h}{2}\right)+\frac{2 b}{3} \sin \left(\frac{3 \omega h}{2}\right)+\frac{2 c}{5} \sin \left(\frac{5 \omega h}{2}\right)}{1+2 \alpha \cos (\omega h)+2 \beta \cos (2 \omega h)}
$$

A constrained minimization problem of the same type as in the previous sections was formulated and solved for these symbols.

\section{Approximation of the integration operator}

The design of integration schemes is substantially limited by the stability requirement which renders high order schemes computationally costly. It is well known [5] that an explicit $k^{\text {th }}$ order Runge-Kutta method, with $k>4$, should employ at least $k+1$ stages or function evaluations. This large number of function evaluations makes high order schemes impractical. Therefore, efforts have been made to obtain schemes of lower order with improved characteristics. Within this approach, the free variables in the Runge-Kutta schemes were set to yield better truncation error [5] or extended stability region [6]. A generalization of this idea is to give up on formal accuracy in order to obtain better approximation of the wavenumbers relevant to the problem solved.

The discrete time integration of linear constant coefficient partial differential equation

$$
\frac{\partial u}{\partial t}=L u
$$

amounts to approximation of the exact discrete solution $e^{L^{h}} u_{0}$. Therefore, the integration scheme may be written as

$$
P_{n}\left(L^{h} \Delta t\right)=\sum_{i=0}^{n} a_{i}\left(L^{h} \Delta t\right)^{i}
$$

where $a_{i}$ may depend on $L^{h}$. The order of the integration scheme is determined by the number of first terms $a_{i}$ which agrees with the Taylor expansion of $e^{x}$.

The derivation of the integration schemes is similar to that of derivative discretization, i.e., a constrained quadratic optimization problem is formulated based on the error estimate (2.20). The solution of this minimization problem yields an improved integration scheme. However, the derivation of integration schemes is more involved than the generation of compact schemes since the stability condition leads to a nonlinearly constrained minimization problem. 
Following (2.20) the next optimization problem is defined :

$$
\min _{a_{i}} \int_{-\frac{\pi}{h}}^{\frac{\pi}{h}}\left|e^{L^{h}(\omega h) \Delta t}-P_{n}\left(\hat{L}^{h}(\omega h) \Delta t\right)\right|^{2}|\hat{u}(\omega h)|^{2} d \omega
$$

subject to the constraints

$$
\begin{aligned}
a_{i} & =\frac{1}{i !} & 0 \leq i \leq p \\
\left|\hat{P}_{n}\left(\hat{L}^{h}(\omega h) \Delta t\right)\right|^{2} & \leq 1 & \omega \in\left[-\frac{\pi}{h}, \frac{\pi}{h}\right]
\end{aligned}
$$

where $L^{h}$ is the discrete approximation of $L$ and $p$ is the order of the $n$ stage formula. Condition (4.4) can be treated by substitution, but the stability condition requires an explicit treatment.

In accordance with our general approach, we believe that second order formal accuracy suffices. It remains to determine the number of stages in the integration formula. This should be chosen to assure that the error in space and time discretizations (2.14) and (2.20), respectively, will be of similar magnitude. In the present work five stage schemes of second order were investigated, i.e., $n=5$ and $p=2$. Integration formulas were obtained for optimized seven point tridiagonal compact schemes approximating the first derivative, and were tested for the advection equation in one and two space dimensions.

An important feature of the present approach is that once a feasible minimum has been found for a prescribed initial value and a given CFL number, the generated scheme will be stable for this data. This might enable the use of somewhat larger time steps.

\section{Approximation of differential operators}

The method introduced in the previous sections for generating optimal finite difference approximations for derivatives and time integration schemes for linear constant coefficient equations can be extended to more general equations. These ideas can be easily adapted to handle with similar efficiency more involved problems.

The error bounds derived in Section 2 can be generalized for $d$ dimensional problems; noting that the same proof holds for the $d$ dimensional case after changing the integration over $\left[-\frac{\pi}{h}, \frac{\pi}{h}\right]$ to multi integration over the box $\left[-\frac{\pi}{h}, \frac{\pi}{h}\right]^{d}$. This suggests that approximation of the differential equation should be obtained by solving constrained optimization problems in $d$ dimensional Fourier space for a large set of parameters. For some equations, solving this large minimization problem might be essential to achieve accurate schemes. Quite often, though, a set of simpler minimization problems can be obtained by optimizing each partial derivative separately, resulting in highly accurate approximations.

The approach, which was successfully tested in the present paper, divides the optimization process into two stages. In the first stage a set of schemes are designed for a large enough variety of typical initial data (e.g., Gaussians with different parameters, in our examples). This precomputation is performed once and its results are used in subsequent simulations. In the second stage, the actual simulation, the initial data $u_{0}$ is Fourier transformed to obtain $\hat{u}_{0}$. The discretization of the partial derivatives is determined by approximating $\hat{u}_{0}$ as a product of one dimensional functions for which optimized schemes were designed. Fach partial derivative 
is discretized using the corresponding one dimensional optimized scheme. The time marching scheme is selected from the set of scheme corresponding to the approximating one dimensional functions. In the present work the selection was done by computing the $\mathrm{L}_{2}$ error norm of each candidate integration scheme when applied to the approximate initial data with the already determined discretizations, and selecting the minimum norm scheme. This computation, too, can be done prior to the actual simulation for a large set of approximated initial data. Thus, the marching scheme selection can be done by looking up in a precomputed table. The robustness of the proposed schemes to perturbations in the initial data yields this optimization very efficient; as can be seen in the numerical results presented in Section 6. It should be noted that the time required to obtain an appropriate scheme using this approach is negligible relative to the simulation time.

When the frequencies present in the solution change with time, e.g., due to time dependent source term, the computation of the optimized schemes should be repeated once a large cumulative change has occurred. Still, the relative cost of of this computation is minimal.

The Fourier transform gives the energy content of the whole initial data. It may occur, that the initial data is smooth at some regions of the computational domain and oscillatory in others, in which case the designed approximation will give good performances over the whole domain. One can do better by computing a different scheme for each region and using a weighted sum of the resulting schemes near region boundaries. This requires computing the Fourier transform locally in each region. The localization to a particular region can be achieve by multiplying $\hat{u}_{0}$ by a $C^{\infty}$ function with a compact support which encloses the region.

In some cases, systems of equations may be treated in a similar way. Look first at a one dimensional first order system

$$
\begin{aligned}
u_{t} & =A u_{x} \\
u(x, 0) & =u_{0}(x)
\end{aligned}
$$

where $A$ is a $p \times p$ symmetric matrix. Let $\Lambda=P^{-1} A P$ be a diagonal matrix, and denote $v=P u$. The discretization of the system

$$
\begin{aligned}
v_{t} & =\Lambda v_{x} \\
v(x, 0) & =P u_{0}(x)
\end{aligned}
$$

can be done in an analogous way to the scalar case, except for the time marching scheme which should be chosen from a set of candidate schemes (as for the multidimensional scalar equations). Thus, highly accurate discretization of the system (5.6) can be achieved by first discretizing (5.7) and using the identity $u_{x}=P^{-1} v_{x}$. For systems in higher dimension

$$
\begin{aligned}
u_{t} & =\sum_{i=1}^{n} A_{i} \frac{\partial u}{\partial x_{i}} \\
u(x, 0) & =u_{0}(x)
\end{aligned}
$$

each partial derivative should be optimized separately. In this case, we require that all $A_{i}$ will be symmetric, but it is not necessary that they are simultaneously diagonalizable.

The proposed schemes might be useful for nonlinear equations, as well. There, one should design the schemes for the linearized equation; and will be obliged modify them once a large change in the amplitude of the wavenumbers appearing in the solution occur. 


\section{Numerical results}

\subsection{Approximation of derivatives}

The constrained minimization problem (3.5) for the space discretization can be easily solved by substitution using (3.2). Differentiation of the resulting quadratic form provides a set of necessary conditions holding at the minimum. This nonlinear system can be solved using Newton method, yielding a local minimum. Since the schemes obtained using this process significantly improve previously known schemes [7], no attempts were made to find the other zeroes of the nonlinear system, searching for better minima.

Three types of schemes were studied : (a) tridiagonal with five points stencil, i.e., $\beta=c=0$, (b) tridiagonal with seven points stencil, i.e., $\beta=0$, (c) pentadiagonal with seven points stencil. The initial approximation to the Newton iteration was, typically, a compact scheme with the same structure, taken from [7].

It can be observed, in figures 1 and 5 , that the modulos of symbol of the optimized pentadiagonal scheme for the first and second derivative is larger then the modulus of the differential symbol. This error is exceedingly larger for schemes generated to approximate narrower spectra. The overshooting occurs in the highest end of the spectrum for wavenumbers not appearing in the solution. However, since the stability of a scheme is determined by the values assumed by $L^{h}(\omega h)$ [11], this type of scheme is applicable only with small CFL. Moreover, the desired robustness is limited by this phenomenon. Therefore, this behavior of the approximation can not be ignored. A possible remedy can be found in searching for other minimizers of the quadratic form. Using the tridiagonal scheme as initial approximation for the Newton process converged to solutions without this limiting property but with reduced resolution, similar to the tridiagonal schemes. Other possible directions, e.g., further looking for other minima or penalizing in the cost function for this behavior were not explored. This is since we believe that for practical applications pentadiagonal systems are too costly to solve, whereas the tridiagonal schemes offer similar resolution characteristics, are easier to solve and do not suffer from this deficiency. The pentadiagonal scheme are given mainly for theoretical reasons as a counterpart to the spectral-like approximations.

A proper appreciation of the superiority of the proposed schemes can be gained by integrating with them hyperbolic equations for long time, provided the integration process introduces only negligible numerical errors. This requirement necessitates either using high order integration

schemes or employing exact integration, as was done in the present work. The experiments described in next subsections clearly demonstrate the superior behavior of the proposed optimized schemes.

\subsubsection{Approximation of the first derivative}

Compact finite difference schemes were designed and tested for initial data of the form $e^{-\alpha \omega^{2}}$ for several values of $\alpha$. In Figure 1, the symbols of schemes corresponding to $\alpha=2$ are plotted, as well as the weighted error

$$
\left|L(\omega h)-L^{h}(\omega h)\right||\hat{u}(\omega h)|
$$


for the more accurate schemes. The coefficients of the optimized schemes can be found in Appendix A. The coefficients of the other schemes were taken from [7]. For scheme (a) the coefficients were

$$
\alpha=\frac{1}{3}, \beta=0, a=\frac{14}{9}, b=\frac{1}{9}, c=0
$$

The coefficients of scheme (c) were

$$
\alpha=\frac{3}{8}, \beta=0, a=\frac{25}{16}, b=\frac{1}{5}, c=-\frac{1}{80}
$$

The coefficients of the spectral-like scheme (e) were

$$
\alpha=0.5771439, \beta=0.0896406, a=1.3025166, b=0.99355, c=0.03750245
$$

It can be seen that each optimized scheme better approximates the differential operator than its non-optimized counterpart. In Figure 1, one can observe that although the symbol of the spectral-like pentadiagonal scheme follows the differential symbol for more wavenumbers than the tridiagonal scheme, the $\mathrm{L}_{2}$ norm of truncation error of tridiagonal scheme is somewhat smaller for this data. This can be explained by noting that the error of the tridiagonal scheme is mainly in the high frequencies while the spectral-like scheme has large error at the smoother Fourier components where the present initial data has more energy. The spectral-like scheme attains better resolution at the expense of larger error in lower frequencies. The error in the optimized schemes is significantly smaller than in their counterparts. More precisely, computing the error norms reveals that the error in the tridiagonal scheme is about six times larger than in the optimized tridiagonal scheme while error norm of the spectral-like scheme is about seventeen times larger than in the optimized pentadiagonal. The plot of the absolute value of the error also reveals that the $\mathrm{L}_{2}$ norm was used as a minimization criteria. This can be seen from the several sign changes of the error of the optimized schemes, being in accordance with the averaging property of the chosen norm.

Figure 2 demonstrates the better resolution of the optimized scheme by exact integrating in Fourier space on a 32 points grid with the pentadiagonal spectral-like scheme and the pentadiagonal optimized scheme the equation

$$
\frac{\partial U}{\partial t}=\frac{\partial U}{\partial x}
$$

$\sigma=0.8$ ( $\sigma$ being the CFL number) was used. It is shown that at time $T=10000$, the error in the solution using the optimized scheme is smaller than the error at time $T=1000$ when using the spectral-like scheme. This suggests that the optimized scheme can be used for integration time at least ten times longer than the spectral-like scheme, in close accordance with the ratio of the error norms.

Figure 3 displays the scheme's robustness to perturbation in initial condition. The solution integrated with the optimized scheme far better approximates the exact solution than the one employing the spectral-like approximation, even for initial data different from the one it was designed to resolve. This holds for both smoother and more oscillatory initial data. Although those examples do not give a quantitative view on the relative efficiency of the schemes for those initial data, one can see in both figures that by the time the solution with the optimized scheme developed significant error the error in the one corresponding to the spectral-like scheme is so large it no longer approximates the exact solution. 
Figure 4 shows a two dimensional equation which demonstrates the robustness of the proposed schemes. In this example the initial data was taken to be the Gaussian $e^{-\left(\omega_{1}^{2}+5 \omega_{2}^{2}\right)}$ rotated at an angle of $\frac{\pi}{4}$. Then the program searched for initial data of the form $e^{-\left(n_{1} \omega_{1}^{2}+n_{2} \omega_{2}^{2}\right)}$, for the integers $1 \leq n_{1}, n_{2} \leq 7$ which yielded the best approximation to the initial data. The pentadiagonal schemes optimized for initial data $e^{-n_{1} \omega^{2}}$ and $e^{-n_{2} \omega^{2}}$ were then used to compute $u_{x}$ and $u_{y}$, respectively. In this example $n_{1}=3$ and $n_{2}=2$. The resulting semi discrete system was solved by exact integration in Fourier space on a $32 \times 32$ grid. The plot shows a cut through the solution in the $x$ direction containing the maximum point of the solution. While the solution corresponding to the optimized disretization closely approximates the exact solution, the solution discretized with the spectral-like scheme bears very little resemblance to the exact solution.

\subsubsection{Approximation of the second derivative}

The coefficients of compact schemes for various initial conditions of the form $e^{-\alpha \omega^{2}}$ can be found in Appendix B.

Figure 5 plots absolute value of the symbol of the second derivative and the weighted error, for $\alpha=2$. The parameters of the optimized schemes can be found in Appendix B. The coefficients of the other schemes were taken from [7]. Scheme (a) is given by :

$$
\alpha=\frac{2}{11}, \beta=0, a=\frac{12}{11}, b=\frac{3}{11}, c=0
$$

The coefficients of scheme (c) are :

$$
\alpha=\frac{9}{38}, \beta=0, a=\frac{696-1191 \alpha}{428}, b=\frac{2454 \alpha-294}{535}, c=-\frac{1179 \alpha-344}{2140}
$$

The coefficients of the spectral-like pentadiagonal scheme (e) are :

$$
\alpha=0.50209266, \beta=0.05669169, a=0.21564935, b=1.723322, c=0.1765973
$$

It can be seen that the error in the non optimized schemes is significantly larger than in the optimized ones. It is interesting to note that, again, for this specific data the $\mathrm{L}_{2}$ error norm of the spectral-like scheme is about an order of magnitude larger than the non optimized tridiagonal scheme. This phenomenon suggests that the resolution efficiency is a poor estimate for discretizations evaluation. Computing the error norms reveals that the error in the optimized tridiagonal scheme is about seven times smaller than in the non optimized scheme, whereas the error in the optimized pentadiagonal scheme is seventy times smaller than the spectral-like scheme, for this given data.

The efficiency of the pentadiagonal schemes was compared by integrating the wave equation :

$$
\frac{\partial^{2} U}{\partial t^{2}}=\frac{\partial^{2} U}{\partial x^{2}}
$$

This equation was put in a system form :

$$
\left(\begin{array}{l}
u \\
v
\end{array}\right)_{t}=\left(\begin{array}{cc}
0 & 1 \\
\frac{\partial^{2}}{\partial x^{2}} & 0
\end{array}\right)\left(\begin{array}{l}
u \\
v
\end{array}\right)
$$


This system was exact integrated on a 32 points grid and the results are given in Figures 6-7 demonstrating the improved accuracy of the optimized scheme and its robustness, respectively. Figure 6 demonstrates that the optimized scheme can be used for integration time 37 times, or more, longer than the spectral-like scheme. In Figure 7 the scheme robustness is clearly shown for initial data smoother or more oscillatory than the data for which the scheme was designed. In both cases, by the time a significant error occurs in the solution discretized with the optimized scheme the solution corresponding to the spectral like scheme totally differs from the exact solution.

The initial solution and its approximation, for the two dimensional problem in Figure 8 were obtained similarly to those of the example in Figure 4. While the solution integrated with the optimized scheme closely approximates the exact solution, it is hard to see that the solution corresponding to the spectral-like scheme indeed approximates the same problem.

\subsubsection{Mid cell approximation of the first derivative}

Appendix $\mathrm{C}$ lists the coefficients of schemes designed for various initial data. The coefficients of the schemes taken from [7] are listed below. Scheme (a) is given by :

$$
\alpha=\frac{9}{62}, \beta=0, a=\frac{3}{8}(3-2 \alpha), b=\frac{1}{8}(22 \alpha-1), c=0
$$

The coefficients of scheme (c) are :

$$
\alpha=\frac{75}{354}, \beta=0, a=\frac{37950-39725 \alpha}{31368}, b=\frac{65115 \alpha-3350}{20912}, c=\frac{25669 \alpha-6114}{62736}
$$

The coefficients of the tenth order pentadiagonal scheme (f) are :

$$
\alpha=\frac{96850}{288529}, \beta=\frac{9675}{577058}, a=\frac{683425}{865587}, b=\frac{505175}{577058}, c=\frac{69049}{11731174}
$$

The standard compact schemes give very good resolution in this form (see Figure 9), thus, the improvement introduced by the optimized schemes is smaller. Optimizing the tridiagonal scheme yields a 6.5 smaller error norm while optimizing the pentadiagonal scheme yields a 2.5 times smaller norm. In this case, the error norm of the optimized tridiagonal scheme is very close to that of the non optimized pentadiagonal scheme.

An interesting option suggested by this approach was to optimize the $\frac{d}{d x}$ operator in order to

get the best approximation for $\frac{d^{2}}{d x^{2}}$, for given initial values. This has been done for the tridiagonal scheme which was used to integrate equation (6.17). It was compared, in Figure 10, to the tridiagonal scheme from [7] where both are used to approximate the second derivative in solving the one dimensional wave equation in the system form (6.18). Again, the optimized scheme gives significantly better approximation.

\subsection{Approximate time integration}

The constrained minimization (4.3)-(4.5) was solved by requiring that the solution will touch the stability constraint at one point while maintaining global stability and and minimizing the functional. The point which gives best result was found by exhaustive search. This straightforward approach yielded the local minima reported in this paper. Somewhat better integration schemes might be achieved by using more advanced optimization techniques [8]. 
According to the general approach outlined in Section 5, one should choose an integration scheme which yields truncation error of similar magnitude in time and space. Since the stability region for several fifth order six stages explicit Runge-Kutta schemes intersects the imaginary axis only in a small neighborhood of the origin $[5,6]$ disabling time marching with large CFL, the optimized scheme was compared with the four stage fourth order Runge-Kutta. We preferred this five stage scheme which has an error norm about five times larger than the space discretization to the seventh order scheme which yields an error norm about eleven times smaller than the space discretization because of its lower computational cost.

The analysis performed in Section 2 suggests that the integration operator should be optimized with respect to the spatial discrete operator employed, i.e., to minimize $\| P\left(L^{h}(\omega h) \Delta t\right)-$ $e^{L^{h}(\omega h) \Delta t} \|_{L_{2}}$ In the following examples $L^{h}$ is the tridiagonal approximation for $\frac{d}{d x}$, when initial data is $e^{-2 \omega^{2}}$ and $\sigma=0.8$. Appendix $D$ contains the coefficients of integration schemes for various initial data when $L^{h}$ is the tridiagonal scheme optimized for the same initial data and $\sigma=0.8$.

Figure 11 plots the real and imaginary parts of $e^{L^{h}(\omega h) \Delta t}$ versus the four stage fourth order Runge-Kutta and the improved scheme. The norm of the imaginary part of the error was reduced by a factor of 31 while its real part was reduced by merely a factor of 2.3 .

Figures 12-13 shows the integration of the advection equation with those scheme on a 32 points grid, demonstrating the superior efficiency and robustness of the proposed schemes. In Figure 12 one can see that the optimized scheme can be used for at least three times longer integration time than the Runge-Kutta scheme applied to the tridiagonal scheme from [7]. The computed error norms suggests the time marching error is dominant in all examples.

The two dimensional example in Figure 14 summarizes the approach suggested in this work. It compares the optimized tridiagonal scheme combined with the appropriate integration formula, to fourth order Runge Kutta applied to non-optimized tridiagonal discretization. Although the analysis in Section 2 applies only to constant coefficient problems, this example shows it holds, heuristicly, to variable coefficient equations, as well. The initial data for this problem was obtained in a similar manner to that in example 4. However, instead of comparing the solutions computed on the $32 \times 32$ grid to the exact solution, they are compared to the solution on a $64 \times 64$ grid which was integrated with the optimized scheme designed for the narrowest computed Gaussian $(\alpha=7)$. The initial data for the finer grid was obtained by bilinear interpolation from the coarser grid. It can be seen that the optimized scheme yields significantly more accurate solution.

\section{Conclusion}

A simple and general approach for the design of finite difference approximation of derivatives and integration formulas was introduced. It was used to design compact finite difference schemes for derivatives evaluation; and the resulting schemes were compared to previously known similar schemes. The guiding line has been to improve the representation of the range of wavenumbers appearing in the physical problem being solved, taking into account their relative amplitudes. This lead to an $\mathrm{L}_{2}$ measure of the approximation. The resulting schemes combined adaptivity to the specific initial data by the nature of their design and robustness to perturbations in the initial data. The improved resolution had been demonstrated for several problems.

A similar approach was used to design improved integration schemes, taking into account the spatial discretization as well as the initial data. 
The approach suggested in this paper for optimizing discrete operators can be similarly applied to higher derivatives. Its applicability to more general and complex operators should be further investigated.

The use of these ideas to design boundary conditions will be presented elsewhere. 


\section{A Coefficients of first derivative approximations for various ini- tial conditions}

\begin{tabular}{|l||c|}
\hline \hline$\hat{u}_{0}$ & schemes with $\beta=c=0$ \\
\hline \hline$e^{-\omega^{2}}$ & $\alpha=0.3793894912, a=1.575573790, b=0.1832051925$ \\
\hline$e^{-2 \omega^{2}}$ & $\alpha=0.3534620453, a=1.566965775, b=0.1399583152$ \\
\hline$e^{-3 \omega^{2}}$ & $\alpha=0.3461890571, a=1.5633098070, b=0.1290683071$ \\
\hline$e^{-4 \omega^{2}}$ & $\alpha=0.3427812069, a=1.5614141543, b=0.124148259$ \\
\hline$e^{-5 \omega^{2}}$ & $\alpha=0.3408027739, a=1.5602604992, b=0.121345048$ \\
\hline$e^{-6 \omega^{2}}$ & $\alpha=0.3395099051, a=1.5594855939, b=0.119534216$ \\
\hline$e^{-7 \omega^{2}}$ & $\alpha=0.3385987444, a=1.5589295176, b=0.1182679712$ \\
\hline
\end{tabular}

\begin{tabular}{|l||c|}
\hline \hline$\hat{u}_{0}$ & schemes with $\beta=0$ \\
\hline \hline$e^{-\omega^{2}}$ & $\alpha=0.4303030674, a=1.5567577428, b=0.3451622238, c=-0.0413138317$ \\
\hline$e^{-2 \omega^{2}}$ & $\alpha=0.3991476265, a=1.5636386371, b=0.2563784492, c=-0.0217218334$ \\
\hline$e^{-3 \omega^{2}}$ & $\alpha=0.3904091387, a=1.5638887738, b=0.2348222711, c=-0.0178927675$ \\
\hline$e^{-4 \omega^{2}}$ & $\alpha=0.3863287472, a=1.5637497712, b=0.2252138483, c=-0.0163061252$ \\
\hline$e^{-5 \omega^{2}}$ & $\alpha=0.3839604005, a=1.5635937780, b=0.21976694619, c=-0.0154399233$ \\
\hline$e^{-6 \omega^{2}}$ & $\alpha=0.3824122042, a=1.5634617985, b=0.21625718276, c=-0.0148945794$ \\
\hline$e^{-7 \omega^{2}}$ & $\alpha=0.3813206436, a=1.5633544597, b=0.21380659696, c=-0.0145197694$ \\
\hline \hline
\end{tabular}

\begin{tabular}{|l||c|}
\hline \hline$\hat{u}_{0}$ & general schemes \\
\hline \hline$e^{-\omega^{2}}$ & $\alpha=0.5779403671, \beta=0.0890143475$ \\
& $a=1.3030269541, b=0.994883769, c=0.0359987066$ \\
\hline$e^{-2 \omega^{2}}$ & $\alpha=0.5801818925, \beta=0.0877284887$ \\
& $a=1.3058941939, b=0.9975884963, c=0.0323380724$ \\
\hline$e^{-3 \omega^{2}}$ & $\alpha=0.5821143744, \beta=0.0867224075$ \\
& $a=1.3086733956, b=0.9990906893, c=0.0299094788$ \\
\hline$e^{-4 \omega^{2}}$ & $\alpha=0.5831688320, \beta=0.0862000893$ \\
& $a=1.3102698137, b=0.9997174262, c=0.0287506026$ \\
\hline$e^{-5 \omega^{2}}$ & $\alpha=0.5838221871, \beta=0.0858844217$ \\
& $a=1.3112828763, b=1.0000513827, c=0.0280789585$ \\
\hline$e^{-6 \omega^{2}}$ & $\alpha=0.58426518608, \beta=0.0856735831$ \\
& $a=1.31197935750, b=1.00025665126, c=0.02764152958$ \\
\hline$e^{-7 \omega^{2}}$ & $\alpha=0.58458494112, \beta=0.08552292859$ \\
& $a=1.31248665912, b=1.00039487751, c=0.02733420278$ \\
\hline \hline
\end{tabular}




\section{B Coefficients of second derivative approximations for various initial conditions}

\begin{tabular}{|l||c|}
\hline \hline$\hat{u}_{0}$ & schemes with $\beta=c=0$ \\
\hline \hline$e^{-\omega^{2}}$ & $\alpha=0.2285657609, a=1.0139538409, b=0.4431776810$ \\
\hline$e^{-2 \omega^{2}}$ & $\alpha=0.2028150072, a=1.0598135170, b=0.3458164974$ \\
\hline$e^{-3 \omega^{2}}$ & $\alpha=0.1952770765, a=1.0716695072, b=0.3188846458$ \\
\hline$e^{-4 \omega^{2}}$ & $\alpha=0.1917151916, a=1.0770076313, b=0.3064227519$ \\
\hline$e^{-5 \omega^{2}}$ & $\alpha=0.1896428309, a=1.0800332355, b=0.29925242633$ \\
\hline$e^{-6 \omega^{2}}$ & $\alpha=0.18828772017, a=1.0819792783, b=0.29459616204$ \\
\hline$e^{-7 \omega^{2}}$ & $\alpha=0.18733255632, a=1.0833354275, b=0.29132968512$ \\
\hline
\end{tabular}

\begin{tabular}{|l||c|}
\hline \hline$\hat{u}_{0}$ & schemes with $\beta=0$ \\
\hline \hline$e^{-\omega^{2}}$ & $\alpha=0.3125176074, a=0.7701351999, b=0.9469577413, c=-0.0920577265$ \\
\hline$e^{-2 \omega^{2}}$ & $\alpha=0.2702488609, a=0.8863525584, b=0.7065172637, c=-0.0523721002$ \\
\hline$e^{-3 \omega^{2}}$ & $\alpha=0.2580699154, a=0.9170322739, b=0.6425330979, c=-0.0434255409$ \\
\hline$e^{-4 \omega^{2}}$ & $\alpha=0.2523894606, a=0.9308701065, b=0.6135153110, c=-0.0396064963$ \\
\hline$e^{-5 \omega^{2}}$ & $\alpha=0.2491062584, a=0.9387256232, b=0.5969863585, c=-0.0374994649$ \\
\hline$e^{-6 \omega^{2}}$ & $\alpha=0.2469677390, a=0.9437849227, b=0.5863166347, c=-0.0361660793$ \\
\hline$e^{-7 \omega^{2}}$ & $\alpha=0.2454642305, a=0.9473144209, b=0.5788609571, c=-0.0352469171$ \\
\hline \hline
\end{tabular}

\begin{tabular}{|l||c|}
\hline \hline$\hat{u}_{0}$ & general schemes \\
\hline \hline$e^{-\omega^{2}}$ & $\alpha=0.5024750577, \beta=0.0554440666$ \\
& $a=0.2150536435, b=1.7246523136, c=0.1761322914$ \\
\hline$e^{-2 \omega^{2}}$ & $\alpha=0.5041582074, \beta=0.0527585356$ \\
& $a=0.2120465713, b=1.7488409942, c=0.1529459205$ \\
\hline$e^{-3 \omega^{2}}$ & $\alpha=0.5053986368, \beta=0.0512444502$ \\
& $a=0.2112256102, b=1.7609579037, c=0.1411026601$ \\
\hline$e^{-4 \omega^{2}}$ & $\alpha=0.5061009898, \beta=0.0504756862$ \\
& $a=0.2110263782, b=1.7667867767, c=0.1353401973$ \\
\hline$e^{-5 \omega^{2}}$ & $\alpha=0.5065435817, \beta=0.0500170894$ \\
& $a=0.2109783634, b=1.7701652358, c=0.1319777431$ \\
\hline$e^{-6 \omega^{2}}$ & $\alpha=0.5068465815, \beta=0.0497133535$ \\
& $a=0.2109761550, b=1.7723629924, c=0.1297807226$ \\
\hline$e^{-7 \omega^{2}}$ & $\alpha=0.5070666579 \beta=0.0494975852$ \\
& $a=0.2109890794, b=1.7739051293, c=0.1282342776$ \\
\hline \hline
\end{tabular}




\section{Coefficients of mid cell approximation of the first derivative for various initial conditions}

\begin{tabular}{|l||c|}
\hline \hline$\hat{u}_{0}$ & . schemes with $\beta=c=0$ \\
\hline \hline$e^{-\omega^{2}}$ & $\alpha=0.1824466564, a=0.9847348088, b=0.3801585039$ \\
\hline$e^{-2 \omega^{2}}$ & $\alpha=0.1621215357, a=1.0026558711, b=0.3215872003$ \\
\hline$e^{-3 \omega^{2}}$ & $\alpha=0.1560892225, a=1.0076143702, b=0.3045640747$ \\
\hline$e^{-4 \omega^{2}}$ & $\alpha=0.1532174394, a=1.0099120548, b=0.2965228240$ \\
\hline$e^{-5 \omega^{2}}$ & $\alpha=0.1515399131, a=1.0112348225, b=0.2918450036$ \\
\hline$e^{-6 \omega^{2}}$ & $\alpha=0.1504402935, a=1.0120939889, b=0.2887865980$ \\
\hline$e^{-7 \omega^{2}}$ & $\alpha=0.1496639344, a=1.0126967653, b=0.2866311035$ \\
\hline \hline
\end{tabular}

\begin{tabular}{|l||l|}
\hline \hline$\hat{u}_{0}$ & schemes with $\beta=0$ \\
\hline \hline$e^{-\omega^{2}}$ & $\alpha=0.2803531992, a=0.8656018611, b=0.7202754832, c=-0.0251709460$ \\
\hline$e^{-2 \omega^{2}}$ & $\alpha=0.2421691108, a=0.9108711860, b=0.5897758895, c=-0.0163088538$ \\
\hline$e^{-3 \omega^{2}}$ & $\alpha=0.2311768224, a=0.9233491904, b=0.5531540626, c=-0.0141496081$ \\
\hline$e^{-4 \omega^{2}}$ & $\alpha=0.2260281312, a=0.9290969691, b=0.5361564844, c=-0.0131971911$ \\
\hline$e^{-5 \omega^{2}}$ & $\alpha=0.2230456380, a=0.9323967450, b=0.5263571378, c=-0.0126626068$ \\
\hline$e^{-6 \omega^{2}}$ & $\alpha=0.2211004185, a=0.9345368034, b=0.5199847302, c=-0.0123206966$ \\
\hline$e^{-7 \omega^{2}}$ & $\alpha=0.2197316282, a=0.9360368674, b=0.5155096846, c=-0.0120832956$ \\
\hline \hline
\end{tabular}

\begin{tabular}{|l||c|}
\hline \hline$\hat{u}_{0}$ & general schemes \\
\hline \hline$e^{-\omega^{2}}$ & $\alpha=0.3392424034, \beta=0.0126851467$ \\
& $a=0.7880308119, b=0.8956208871, c=0.0202034010$ \\
\hline$e^{-2 \omega^{2}}$ & $\alpha=0.3364203680, \beta=0.0159838314$ \\
& $a=0.7894607720, b=0.8790559502, c=0.0362916767$ \\
\hline$e^{-3 \omega^{2}}$ & $\alpha=0.3359766282, \beta=0.0164557610$ \\
& $a=0.7895453413, b=0.8768367139, c=0.0384827231$ \\
\hline$e^{-4 \omega^{2}}$ & $\alpha=0.3358345755, \beta=0.0166014190$ \\
& $a 0.78955615727, b=0.87616875736, c=0.03914707436$ \\
\hline$e^{-5 \omega^{2}}$ & $\alpha=0.33577201042, \beta=0.01666433833$ \\
& $a=0.78955722003, b=0.87588406207, c=0.03943141540$ \\
\hline$e^{-6 \omega^{2}}$ & $\alpha=0.33573907328, \beta=0.01669706335$ \\
& $a=0.78955658369, b=0.87573722427, c=0.03957846531$ \\
\hline$e^{-7 \omega^{2}}$ & $\alpha=0.33571963682, \beta=1.67162152050$ \\
& $a=0.78955572985, b=0.87565178238, c=0.03966419181$ \\
\hline \hline
\end{tabular}




\begin{tabular}{|l||c|}
\hline \hline$\hat{u}_{0}$ & schemes with $\beta=0$, designed to approximate $\frac{d^{2}}{d x^{2}}$ \\
\hline \hline$e^{-\omega^{2}}$ & $\alpha=0.2949304593, a=0.8473898079, b=0.7718938474, c=-0.0294227367$ \\
\hline$e^{-2 \omega^{2}}$ & $\alpha=0.2482825125, a=0.9037600128, b=0.6104318128, c=-0.0176268008$ \\
\hline$e^{-3 \omega^{2}}$ & $\alpha=0.2349387889, a=0.9190859222, b=0.5656763757, c=-0.0148847202$ \\
\hline$e^{-4 \omega^{2}}$ & $\alpha=0.2287385754, a=0.9260661646, b=0.5451127700, c=-0.0137017838$ \\
\hline$e^{-5 \omega^{2}}$ & $\alpha=0.2251628777, a=0.9300484196, b=0.5333228985, c=-0.0130455627$ \\
\hline$e^{-6 \omega^{2}}$ & $\alpha=0.2228371850, a=0.9326209622, b=0.5256822919, c=-0.0126288841$ \\
\hline$e^{-7 \omega^{2}}$ & $\alpha=0.2212037269, a=0.9344193325, b=0.52032910793, c=-0.0123409866$ \\
\hline \hline
\end{tabular}

\section{Coefficients of time integration scheme}

\begin{tabular}{|l||c|}
\hline \hline$\hat{u}_{0}$ & third order schemes designed for $\sigma=0.9$ having $a_{0}=1, a_{1}=1, a_{2}=\frac{1}{2}$ \\
\hline \hline$e^{-2 \omega^{2}}$ & $a_{3}=0.166281, a_{4}=0.0397196, a_{5}=0.0076705$ \\
\hline$e^{-3 \omega^{2}}$ & $a_{3}=0.166407, a_{4}=0.0409525, a_{5}=0.0074510$ \\
\hline$e^{-4 \omega^{2}}$ & $a_{3}=0.1664488, a_{4}=0.04111513, a_{5}=0.00739737$ \\
\hline$e^{-5 \omega^{2}}$ & $a_{3}=0.1664805, a_{4}=0.04121264, a_{5}=0.00736302$ \\
\hline$e^{-6 \omega^{2}}$ & $a_{3}=0.1665028, a_{4}=0.04128218, a_{5}=0.00733301$ \\
\hline$e^{-7 \omega^{2}}$ & $a_{3}=0.1665207, a_{4}=0.04133150, a_{5}=0.00731074$ \\
\hline \hline
\end{tabular}




\section{References}

[1] Y. Adam, Highly Accurate Compact Implicit Methods and boundary Conditions, J. Comp. Phys, 24 (1977), pp. 10-22.

[2] R. S. Hirsh, Higher Order Accurate Difference Solutions of Fluid Mechanincs Problems by a Compact Differencing Scheme, J. Comp. Phys., 19 (1975), pp. 90-109.

[3] Z. Kopal, Numerical analysis, 2nd ed., (John Wiley \& Sons, 1961), pp. 552-553.

[4] D. Kishoni and S. Ta'asan, Improved Finite Difference Method for Long Distance Propagation of Waves, Review of Progress in NDE, August 1992, La Jolla California.

[5] L. Lapidus and J. Seinfeld, Numerical Solution of Ordinary Differential Equations, (Academic Press 1971), 298p

[6] J. D. Lawson, An Order Five Runge-Kutta Process with Extended Region of Stability, SIAM J. Numer. Anal., 3 (1966), pp 593-597.

[7] S. K. Lele, Compact Finite Differecne Schemes with Spectral-like Resolution, J. Comp. Phys, 103 (1992), pp. 16-42.

[8] S. McCormick, Nonlinear Programming. Theory, algorithms and applications, (John Wiley \& Sons, 1983), 444pp.

[9] W. L. Miranker, Difference Schemes with Best Possible Truncation Error, Numer. Math., 17 (1971) pp. 124-142.

[10] B. Swartz and B. Wendroff, The Relative Efficiency of Finite Difference and Finite Element Methods. I : Hyperbolic Problems and Splines, SIAM J. Numer. Anal, 11 (1974), pp. 979-993.

[11] R. Vichenevetsy, Stabillity Charts in Numerical Approximation of Partial Differential Equations: A Review, Mathematics and Computers in Simulation, 25 (1979), pp. 170-177.

[12] R. Vichenevetsy and J. B. Bowles, Fourier Analysis of Numerical Approximations of Hyperbolic Equations, (SIAM, 1982), 140pp. 

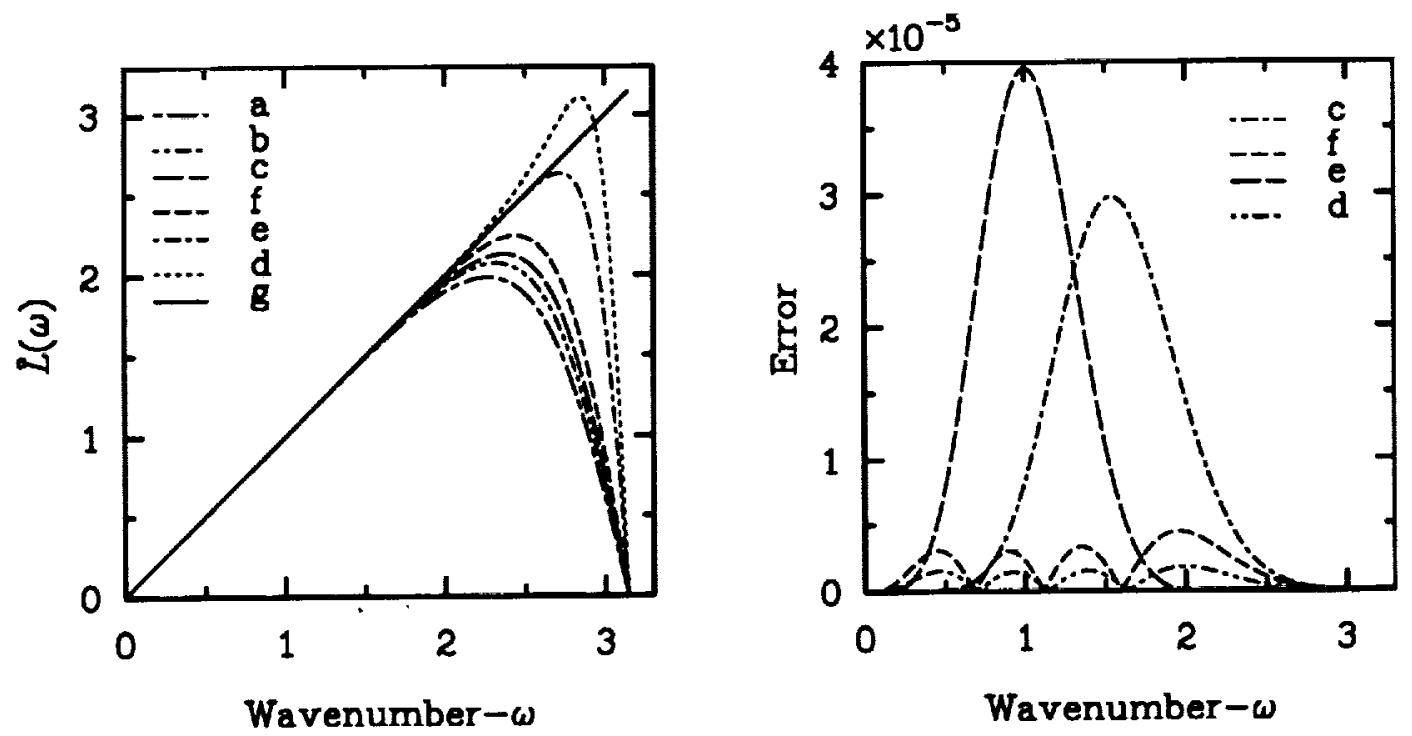

Figure 1: Symbols (left) and absolute value of error (right) for $\frac{d}{d x} \cdot \hat{u}_{0}=e^{-2 \omega^{2}}$. (a) Sixth order tridiagonal scheme $(\beta=c=0)$ (b) Second order optimized tridiagonal scheme $(\beta=c=0)$ (c) Eighth order tridiagonal scheme $(\beta=0)$ (d) Second order optimized tridiagonal scheme $(\beta=0)$ (e) Spectral-like pentadiagonal (f) Optimized pentadiagonal. (g) Exact symbol. Schemes were optimized for $\hat{u}_{0}=e^{-2 \omega^{2}}$.

$$
T=1000 .
$$

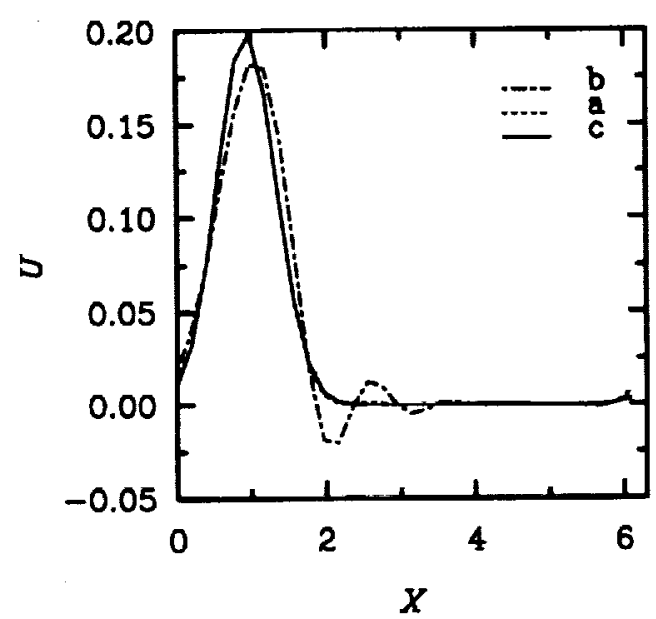

$\mathrm{T}=10000$.

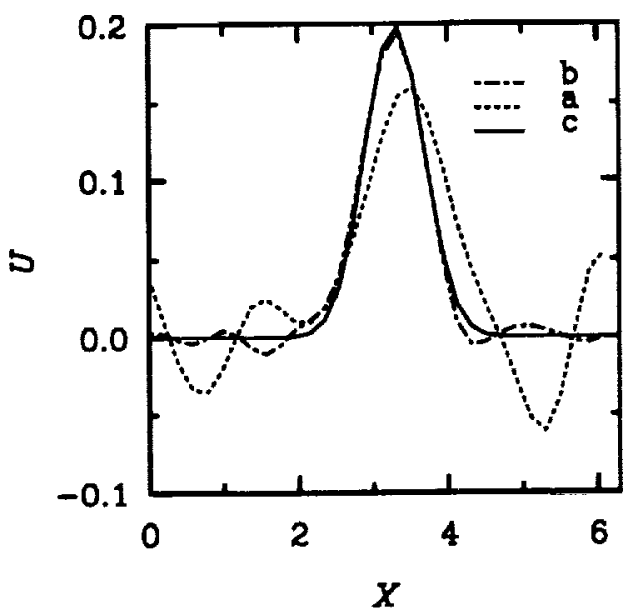

Figure 2: Long time integration of the equation $u_{t}=u_{x}, \hat{u}_{0}=e^{-2 \omega^{2}}, \sigma=0.8$. (a) Pentadiagonal scheme optimized for $\hat{u}_{0}=e^{-2 \omega^{2}}$ (b) Spectral-like pentadiagonal scheme (c) Exact solution. 


$$
T=1800 .
$$

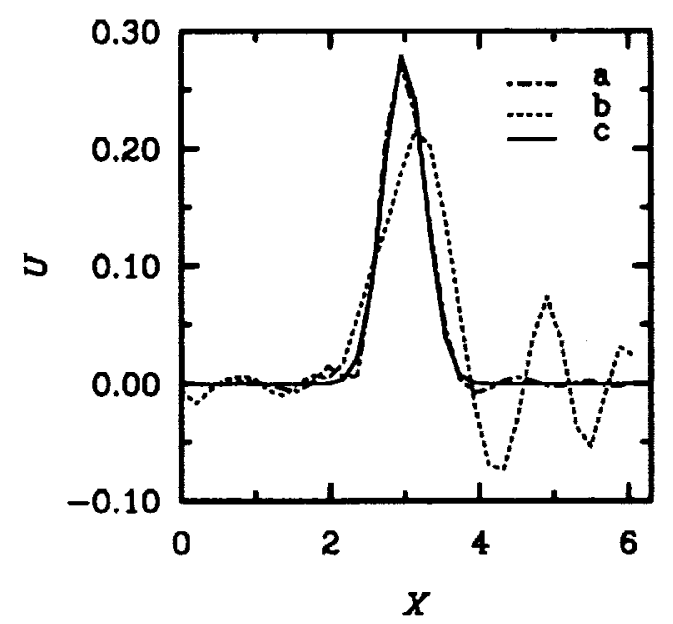

$T=15000$.

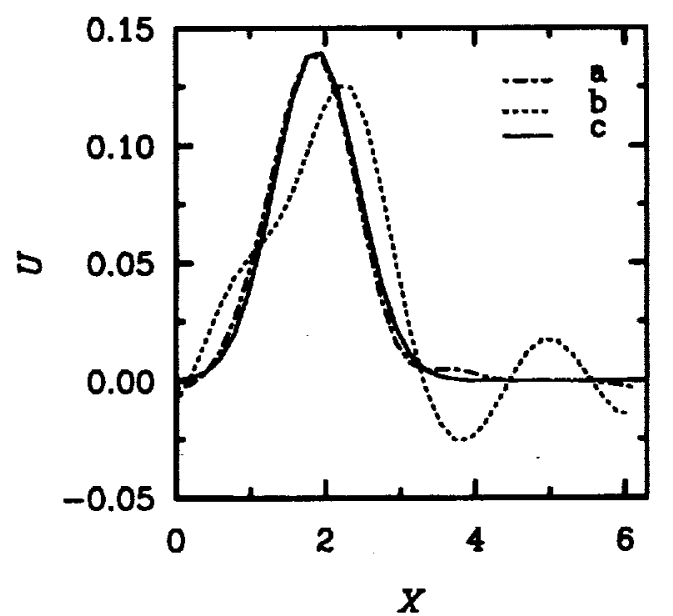

Figure 3: Long time integration of the equation $u_{t}=u_{x}, \sigma=0.8$. Initial solution on the left figure was $\hat{u}_{0}=e^{-\omega^{2}}$; on the right figure it was $\hat{u}_{0}=e^{-4 \omega^{2}}$.

(a) Pentadiagonal scheme optimized for $\hat{u}_{0}=e^{-2 \omega^{2}}$ (b) Spectral-like pentadiagonal scheme (c) Exact solution. 


\section{$\mathrm{T}=6500$.}

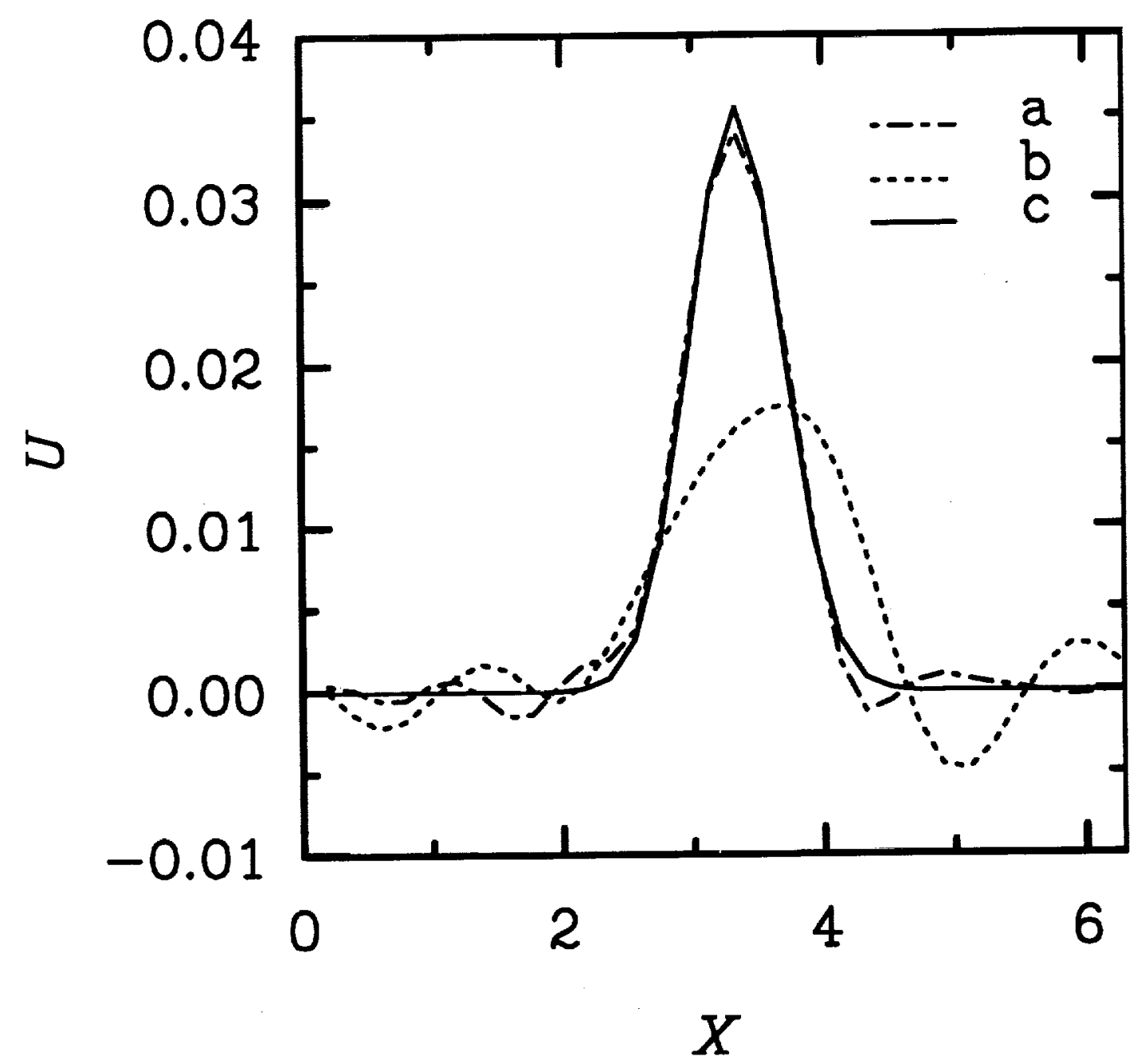

Figure 4: Integration of the equation $u_{t}=u_{x}+u_{y}, \sigma=0.8$ using pentadiagonal schemes. Initial solution was $\hat{u}_{0}=e^{-\left(\omega_{1}^{2}+5 \omega_{2}^{2}\right)}$ rotated at an angle of $\frac{\pi}{4}$. This data was approximated by unrotated gaussian $e^{-\left(3 \omega_{1}^{2}+2 \omega_{2}^{2}\right)}$. (a) Optimized pentadiagonal scheme (b) Spectral-like pentadiagonal scheme (c) Exact solution. 

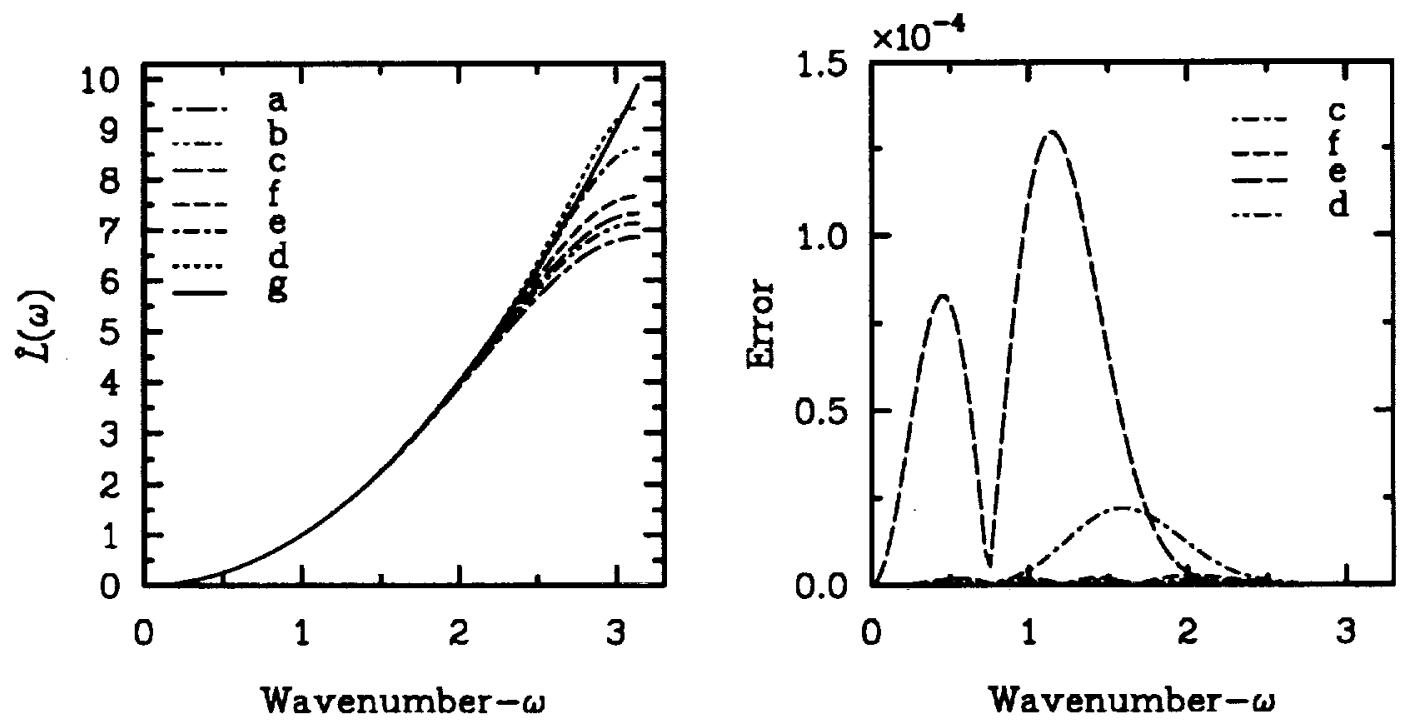

Figure 5: Symbol (left) and absolute value of error (right) for $\frac{d^{2}}{d x^{2}}$. $\hat{u}_{0}=e^{-2 \omega^{2}}$. (a) Sixth order tridiagonal scheme $(\beta=c=0)$ (b) Second order optimized tridiagonal scheme $(\beta=c=0)(c)$ Eighth order tridiagonal scheme $(\beta=0)$ (d) Second order optimized tridiagonal scheme $(\beta=0)$ (e) Spectral-like pentadiagonal (f) Optimized pentadiagonal. (g) Exact symbol. Schemes were optimized for $\hat{u}_{0}=e^{-2 \omega^{2}}$.

$$
\mathrm{T}=200 .
$$

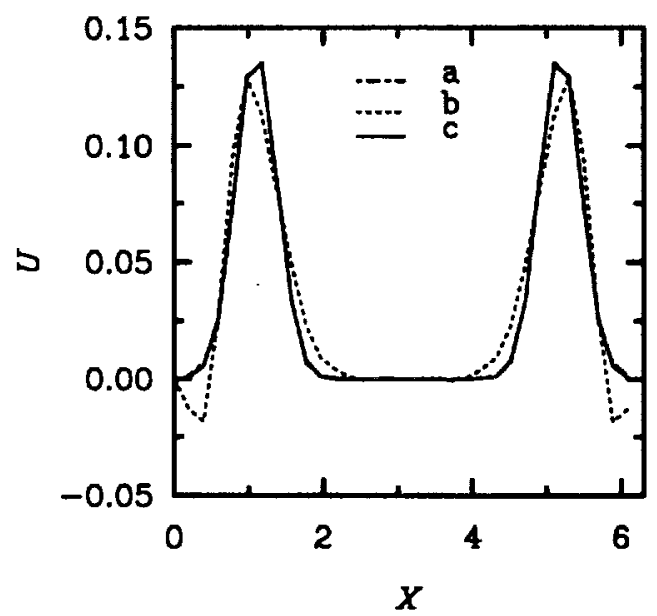

$\mathrm{T}=7500$.

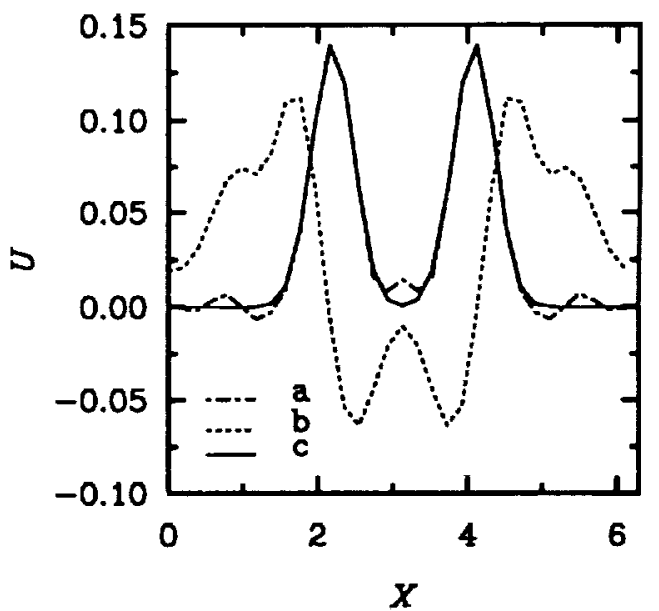

Figure 6: Long time integration for $u_{t t}=u_{x x}, \hat{u}_{0}=e^{-2 \omega^{2}}, \sigma=0.8$. (a) Pentadiagonal scheme optimized for $\hat{u}_{0}=e^{-2 \omega^{2}}$ (b) Spectral-like pentadiagonal scheme (c) Exact solution. 


$$
\mathbf{T}=3200 .
$$

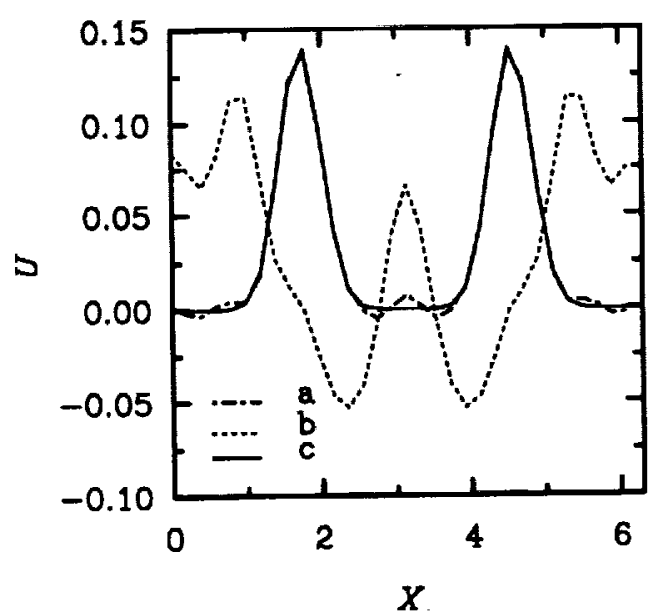

$T=16000$.

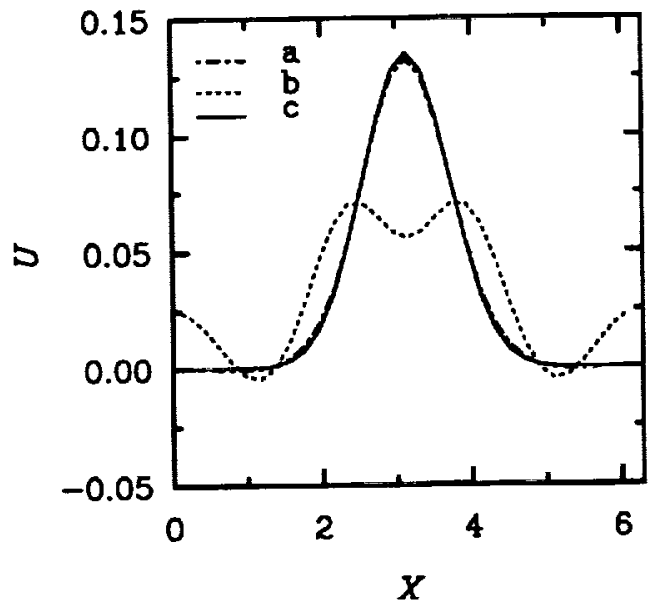

Figure 7: Long time integration for $u_{t t}=u_{x x}, \sigma=0.8$. Initial solution on the left figure was $\hat{u}_{0}=e^{-\omega^{2}}$; on the right figure it was $\hat{u}_{0}=e^{-4 \omega^{2}}$. (a) Pentadiagonal scheme optimized for $\hat{u}_{0}=e^{-2 \omega^{2}}$ (b) Spectral-like pentadiagonal scheme (c) Exact solution. 


$$
\mathrm{T}=6500 .
$$

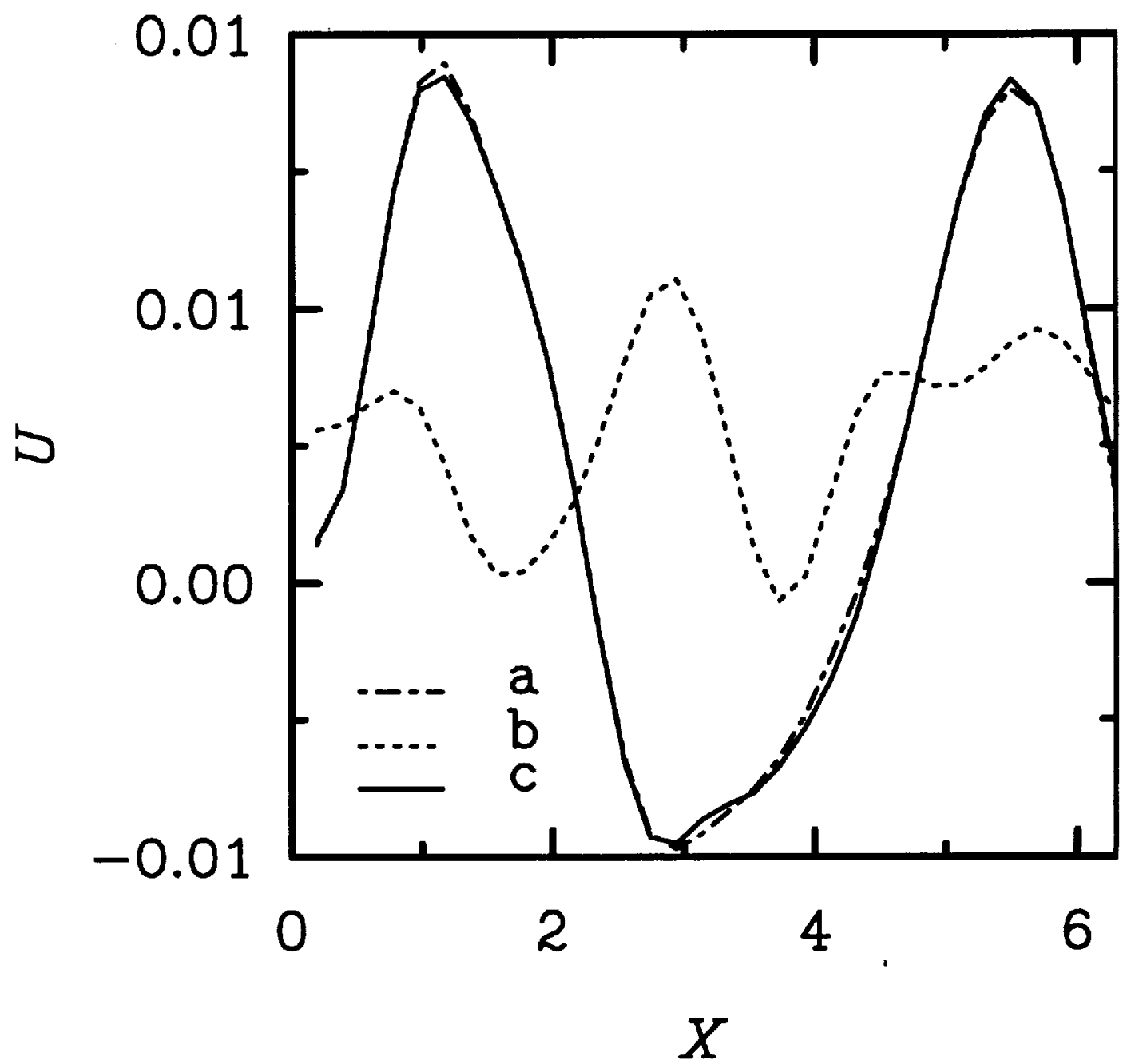

Figure 8: Long time integration for $u_{t t}=u_{x x}+u_{y y}, \sigma=0.8$ using pentadiagonal schemes. Initial solution on the left figure was $\hat{u}_{0}=e^{-\left(\omega_{1}^{2}+5 \omega_{2}^{2}\right)}$ rotated at an angle of $\frac{\pi}{4}$. This data was approximated by unrotated gaussian $e^{-\left(3 \omega_{1}^{2}+2 \omega_{2}^{2}\right)}$. (a) Optimized pentadiagonal scheme (b) Spectral-like pentadiagonal (c) Exact solution. 


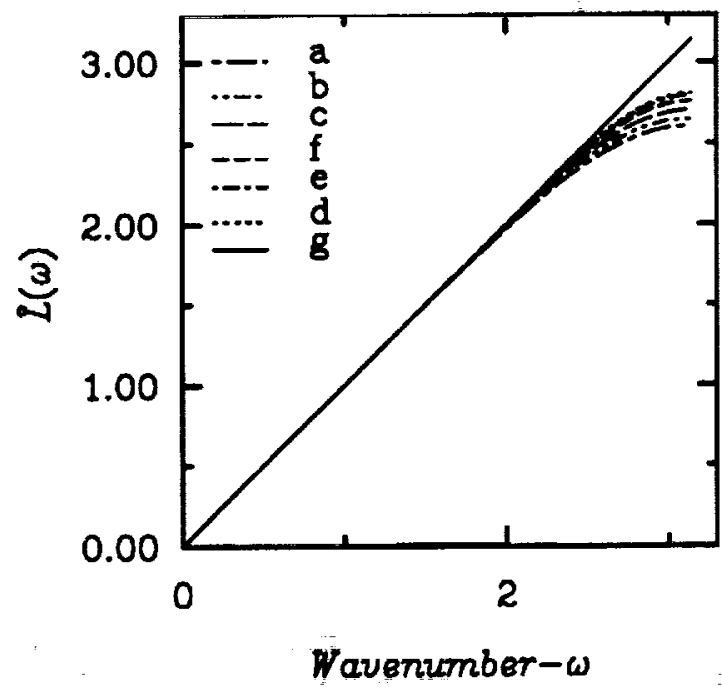

Figure 9: Symbol for mid-cell discretizations of $\frac{d}{d x}, \hat{u}_{0}=e^{-2 \omega^{2}}$. (a) Sixth order tridiagonal scheme $(\beta=c=0)$ (b) Second order optimized tridiagonal scheme $(\beta=c=0)$ (c) Eighth order tridiagonal scheme $(\beta=0)(d)$ Second order optimized tridiagonal scheme $(\beta=0)$ (e) Tenth order pentadiagonal (f) Optimized pentadiagonal. (g) Exact symbol. Schemes were optimized for $\hat{u}_{0}=e^{-2 \omega^{2}}$. 


$$
\mathrm{T}=4500 .
$$

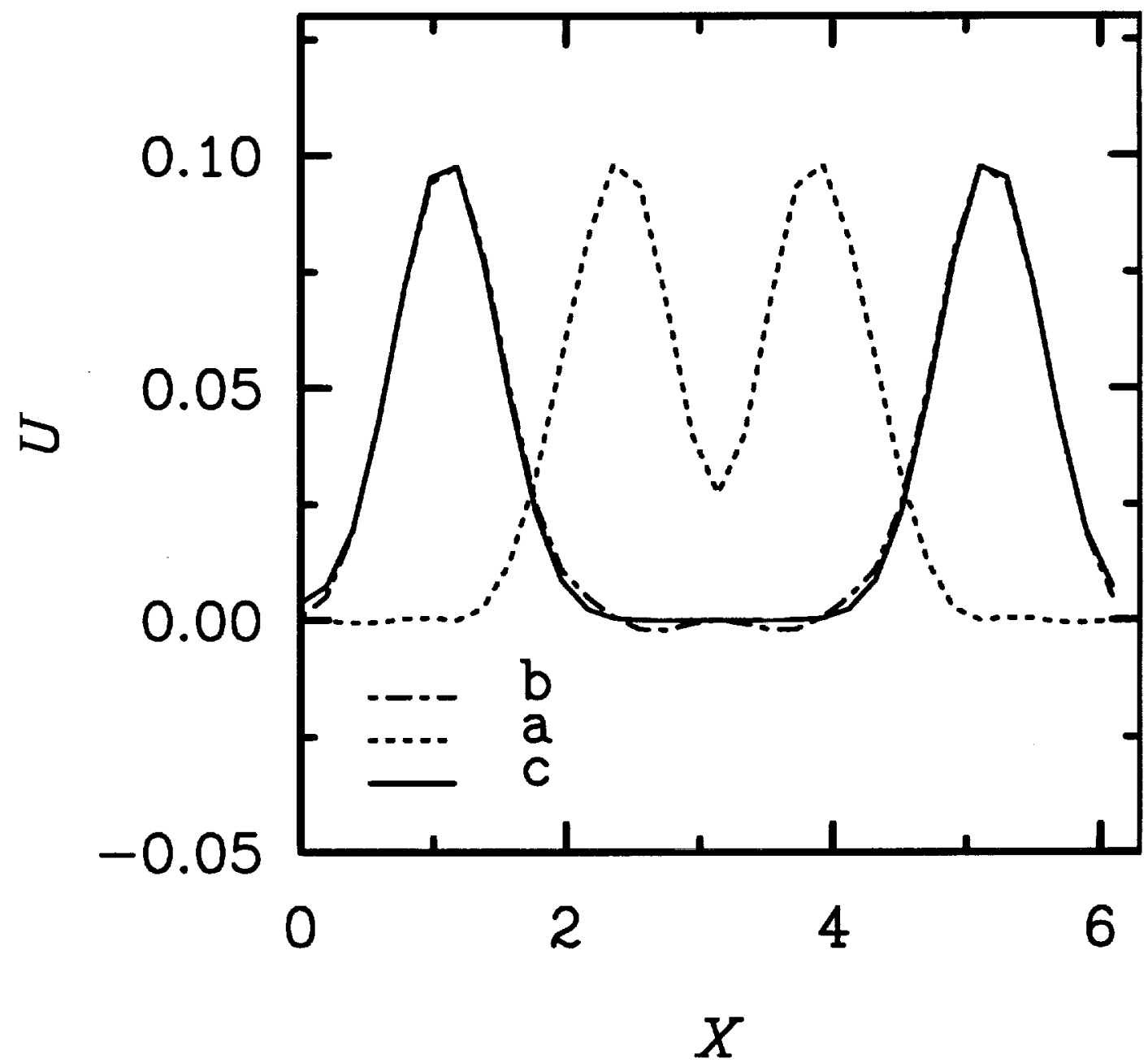

Figure 10: Long time integration for $u_{t t}=u_{x x}, \hat{u}_{0}=e^{-2 \omega^{2}} \sigma=0.8$. (a) Non optimized tridiagonal scheme (b) Tridiagonal mid-cell discretization scheme of $\frac{d}{d x}$ optimized to approximate $\frac{d^{2}}{d x^{2}}$ when $\hat{u}_{0}=e^{-2 \omega^{2}}$ (c) Exact solution. 

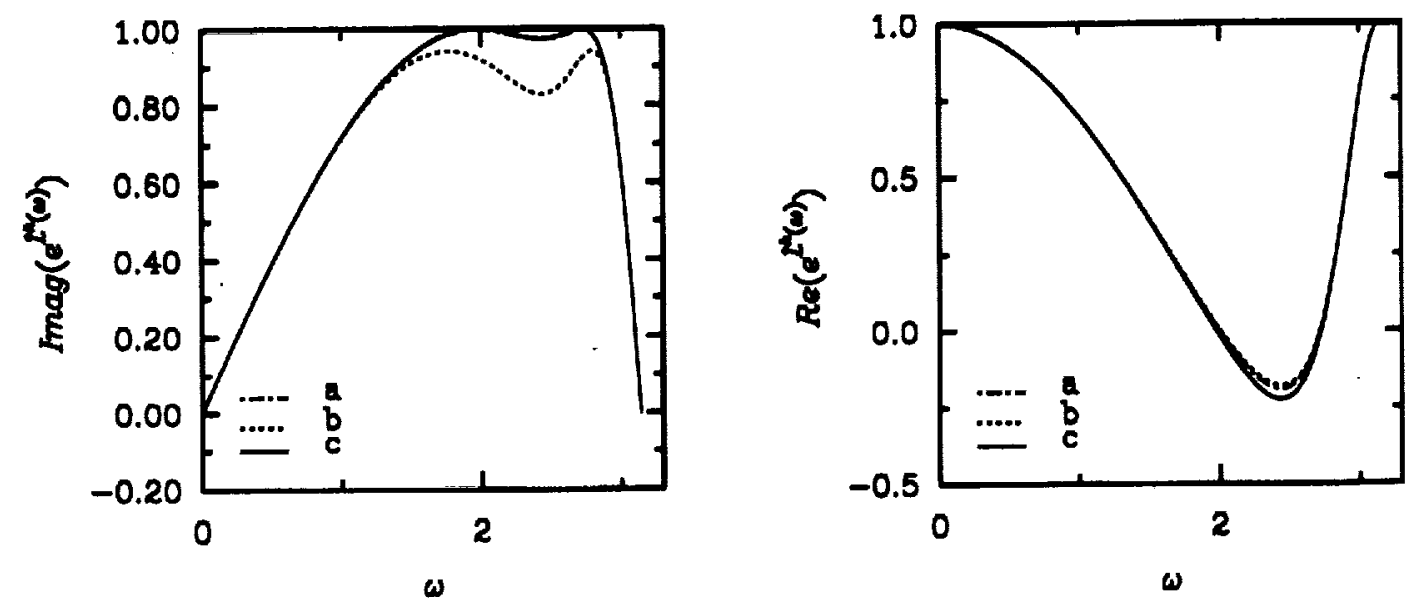

Figure 11: Real and imaginary parts of approximations to $e^{L^{h}(\omega)}$, where $L^{h}(\omega)$ is the symbol of the tridiagonal scheme for $\frac{d}{d x}$ optimized for $\hat{u}_{0}=e^{-2 \omega^{2}}$ and $\sigma=0.8$. (a) Five stage scheme optimized for the same $\sigma$ (b) Fourth order Runge-Kutta (c) Exact time integration.

$T=150$.

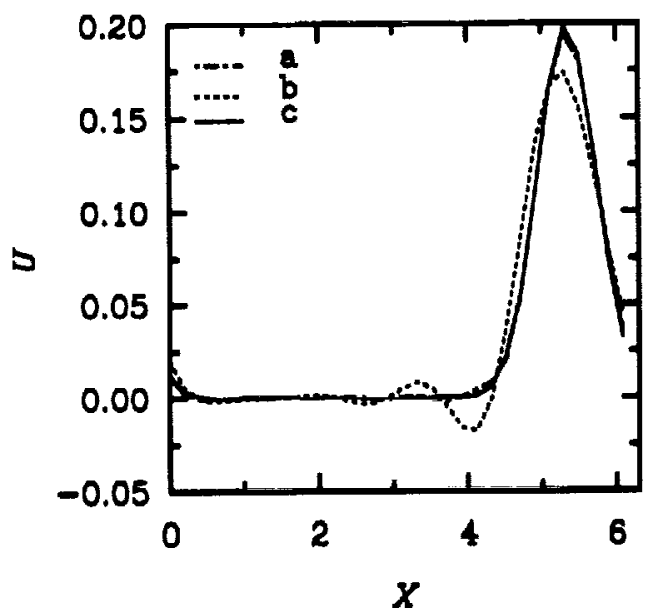

$T=450$.

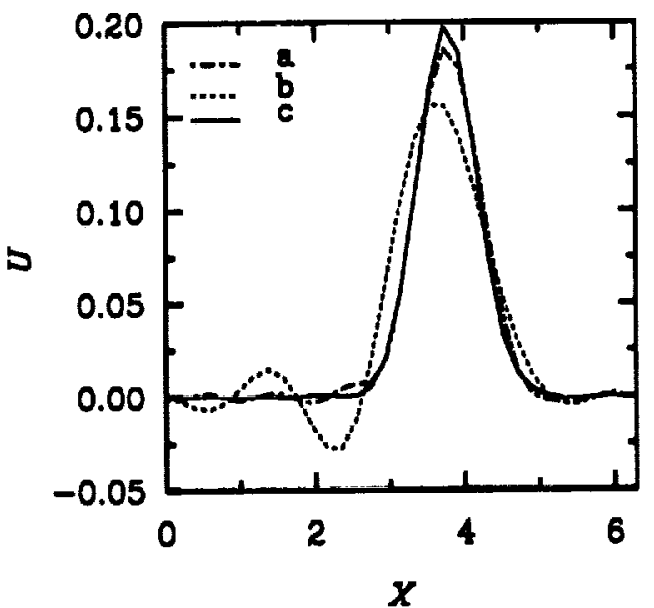

Figure 12: Integration of $u_{t}=u_{x}, \hat{u}_{0}=e^{-2 \omega^{2}}, \sigma=0.8$. The space derivative is computed using the tridiagonal compact scheme optimized for the same initial date and $\sigma$. (a) Five stage scheme optimized for this scheme and CFL (b) Fourth order Runge Kutta (c) Exact time integration 


$$
\mathrm{T}=45 .
$$

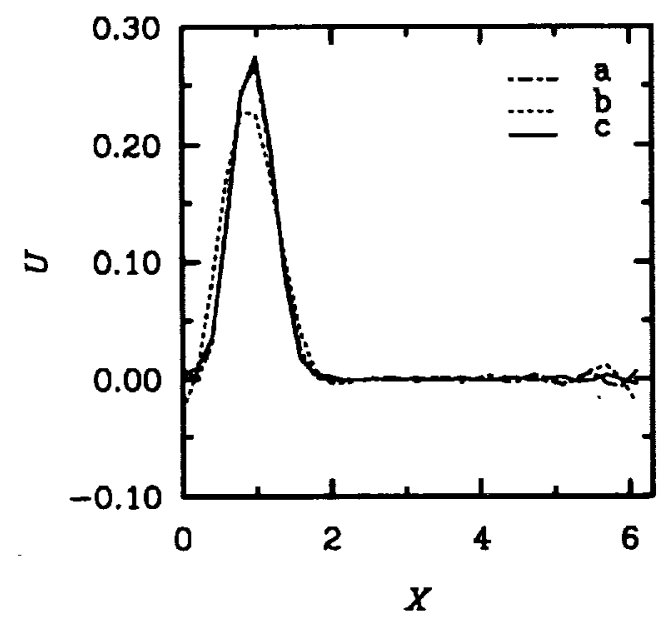

$T=1000$.

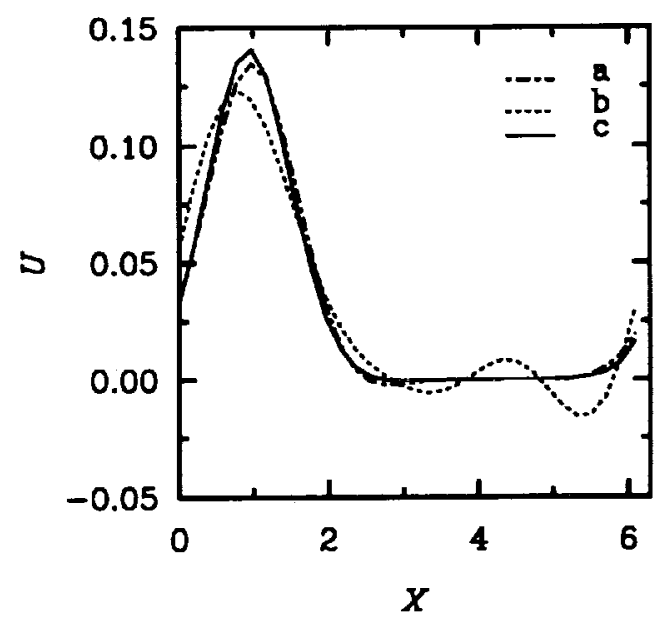

Figure 13: Integration of $u_{t}=u_{x}, \sigma=0.8$. Left: $\hat{u}_{0}=e^{-\omega^{2}} ;$ Right: $\hat{u}_{0}=e^{-4 \omega^{2}}$. (a) Five stage scheme optimized for this scheme and CFL (b) Fourth order Runge Kutta (c) Exact time integration. 


$$
\mathrm{T}=400 .
$$

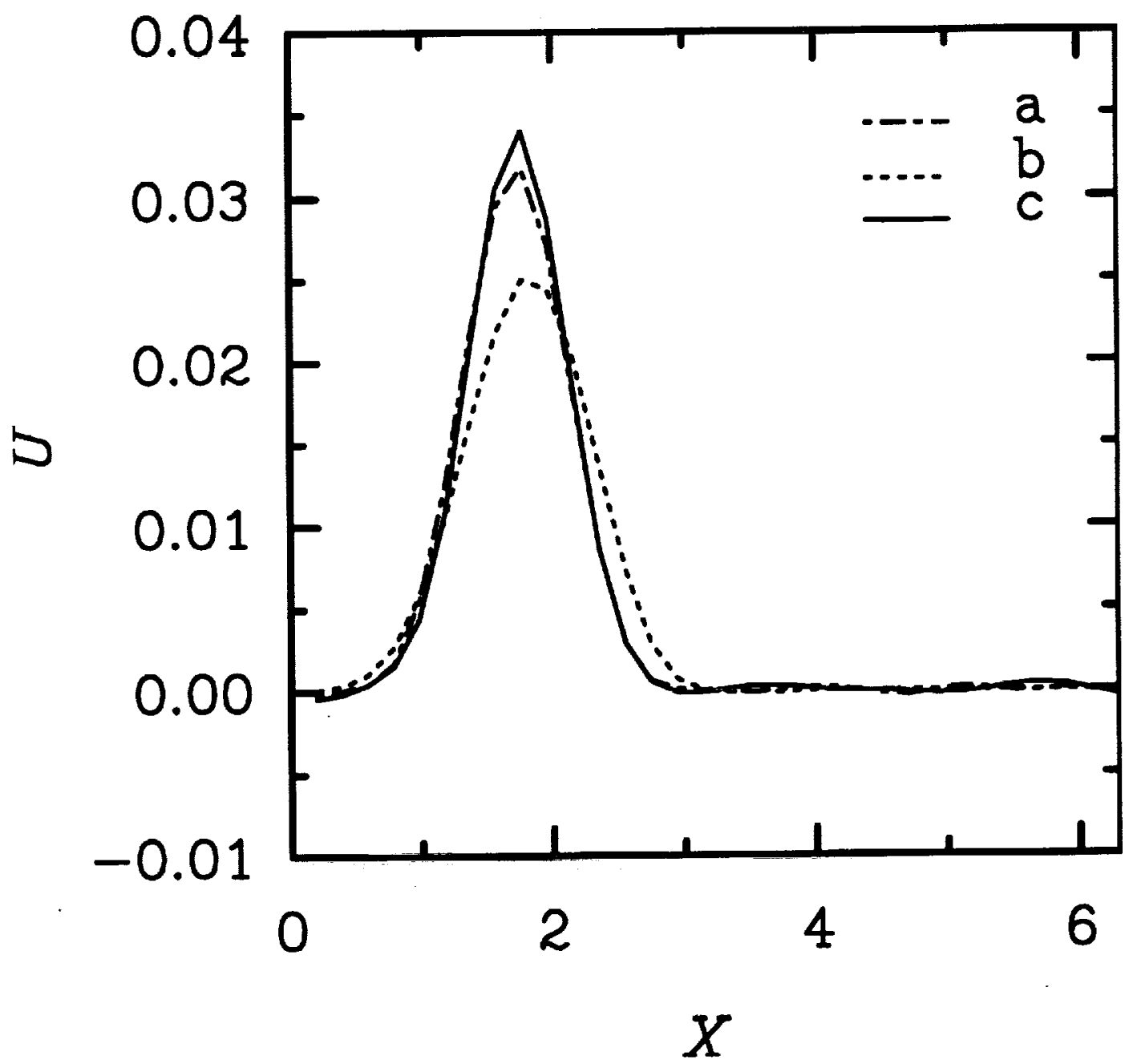

Figure 14: Integration of $u_{t}=u_{x}+0.5(1+0.6 \sin (2 \pi y)) u_{y}, \sigma=0.8$ using tridiagonal schemes. Initial solution was $\hat{u}_{0}=e^{-\left(\omega_{1}^{2}+5 \omega_{2}^{2}\right)}$ rotated at an angle of $\frac{\pi}{4}$. This data was approximated by unrotated gaussian $e^{-\left(3 \omega_{1}^{2}+2 \omega_{2}^{2}\right)}$. (a) Optimized tridiagonal scheme and optimized marching scheme (b) Tridiagonal scheme integrated with fourth order Runge-Kutta. (c) A fine grid solution (practically exact) 


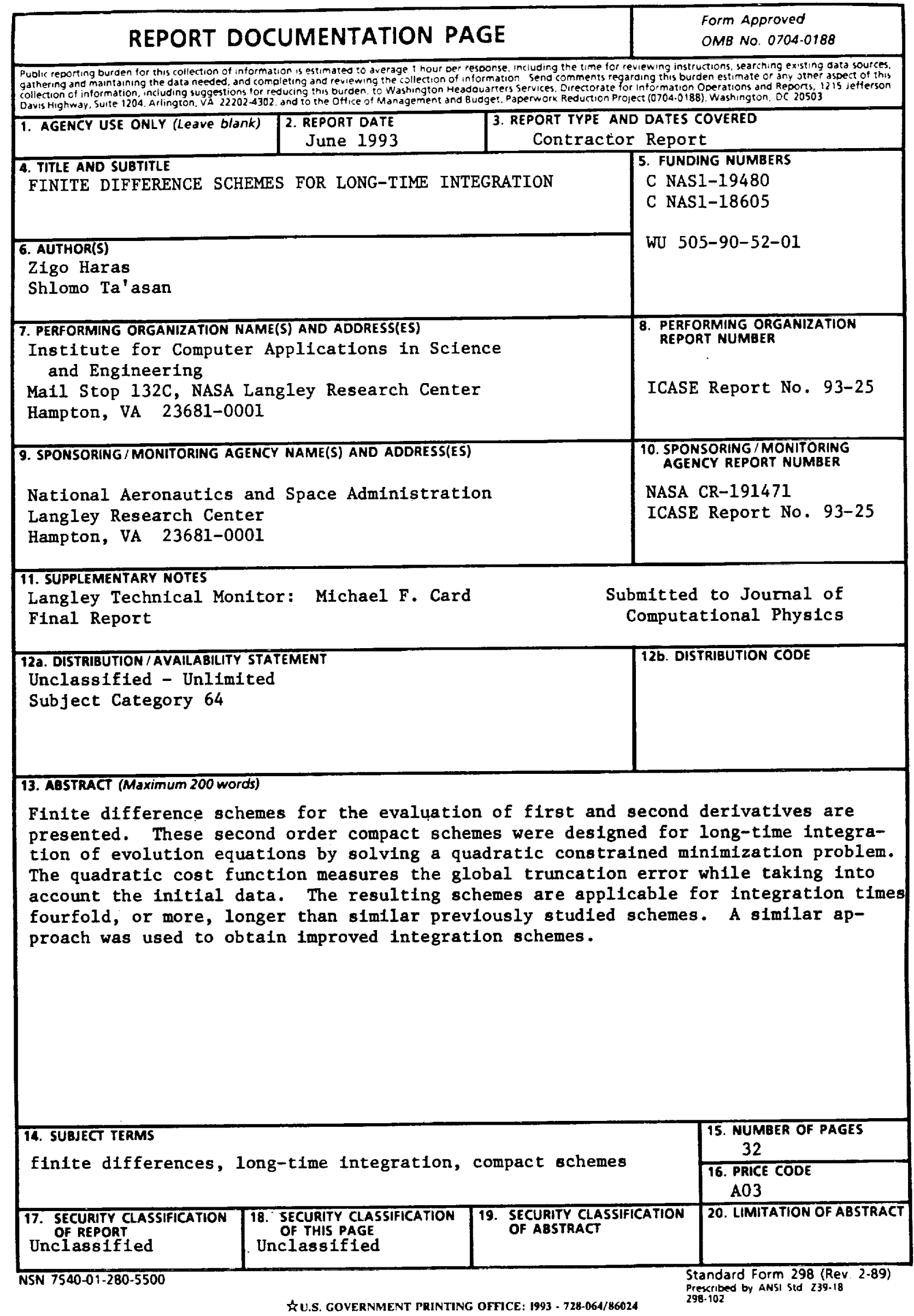




